\title{
Finite-Time Stabilization for a Class of Nonlinear Differential-Algebraic Systems Subject to Disturbance
}

\author{
Xiaohui Mo, ${ }^{1}$ Huawei Niu, ${ }^{2}$ and Qixun $\operatorname{Lan}^{3}$ \\ ${ }^{1}$ School of Computer Engineering, Jinling Institute of Technology, Nanjing 211169, China \\ ${ }^{2}$ School of Mathematics, Pingdingshan Institute of Education, Pingdingshan 467000, China \\ ${ }^{3}$ School of Mathematics and Physics, Henan University of Urban Construction, Pingdingshan 467000, China \\ Correspondence should be addressed to Qixun Lan; q.lan@hncj.edu.cn
}

Received 13 July 2016; Accepted 15 January 2017; Published 15 February 2017

Academic Editor: Rigoberto Medina

Copyright (C) 2017 Xiaohui Mo et al. This is an open access article distributed under the Creative Commons Attribution License, which permits unrestricted use, distribution, and reproduction in any medium, provided the original work is properly cited.

\begin{abstract}
In this paper, finite-time stabilization problem for a class of nonlinear differential-algebraic systems (NDASs) subject to external disturbance is investigated via a composite control manner. A composite finite-time controller (CFTC) is proposed with a threestage design procedure. Firstly, based on the adding a power integrator technique, a finite-time control (FTC) law is explicitly designed for the nominal NDAS by only using differential variables. Then, by using homogeneous system theory, a continuous finite-time disturbance observer (CFTDO) is constructed to estimate the disturbance generated by an exogenous system. Finally, a composite controller which consists of a feedforward compensation part based on CFTDO and the obtained FTC law is proposed. Rigorous analysis demonstrates that not only the proposed composite controller can stabilize the NDAS in finite time, but also the proposed control scheme exhibits nominal performance recovery property. Simulation examples are provided to illustrate the effectiveness of the proposed control approach.
\end{abstract}

\section{Introduction}

Differential-algebraic systems (DASs) [1-3] known as singular systems [4-7], descriptor systems [8-10], or implicit systems [11] represent an important class of systems. Because DASs provide a more general representation than normal systems in the sense of modelling, many practical systems, such as power systems, robot systems, and economic systems, are beyond the description of normal systems but can be described by DASs [4]. Hence, the analysis and control design problems of DASs have attracted a lot of attention from the engineering and academic fields in the past several decades. However, most existing work focus on linear DASs; see [4$6,11-14]$ and the references therein.

Nonlinear DASs (NDASs) characterize a class of rather complex systems, which not only have nonlinearities but also have singular nature of algebraic constraints. Therefore, compared to linear DASs, the investigation of NDASs is more difficult. As far as the control problem for NDASs is concerned, only a few results are available in the literature. Under the assumption that nonlinear differential-algebraic equations can be described by a nonlinear control system on a smooth manifold, the feedback stabilization problem of NDASs was addressed in [15]. For a class of affine nonlinear singular systems, the feedback control problem and exact linearization approach were considered in [7]. By using the feedback linearization approach, the feedback stabilization problem for a class of NDASs was solved in [7]. By linear matrix inequality technique, the work [16] considered the stability and the damping control design problems for a class of NDASs. Based on backstepping technique [17], a robust controller design method was proposed for a class of NDASs. It is known to all that disturbances widely exist in practical control systems and bring negative effects to the control performance of these systems. Disturbance attenuation is of great importance in the control system design [11, 18-23]. In [10], $H_{\infty}$ control problem for a class of nonlinear descriptor systems was studied, and the necessary and sufficient condition was derived for the solvability of the problem. By using Hamiltonian function method, the stabilization and $H_{\infty}$ control problems for a class of NDASs were addressed 
in [24]. For a general class of nonlinear singular systems subjected to external disturbance, the work [25] removed the normalizability assumption and proposed a complete solution of output regulation problems. By using internal model approach, robust output regulation problem for a class of nonlinear singular systems subjected to disturbance was investigated in [26]. In order to enhance the disturbance rejection ability of NDASs, a composite hierarchical antidisturbance control method was proposed for a class of nonlinear singular systems with multiple disturbances in [27]. It should be pointed out that almost all the existing results about the control problem of NDASs mentioned above only concern the asymptotic stability which means convergence with an infinite settling time.

Under a finite-time controller, the closed-loop usually demonstrates not only faster convergence rate but also higher accuracy as well as better disturbance rejection properties [28-31]. In view of these advantages, at present, more and more interest has been focused on the system control and design problems by using FTC technique [32-38]. However, most of the finite-time stabilization results available in the literature are only applicable to normal control systems. Due to the inherent characteristics of NDASs, the finitetime stabilization problems of NDASs are more challenging. Recently, an energy based approach was proposed to study the finite-time stabilization and finite-time $H_{\infty}$ control problems of a class of nonlinear Hamiltonian descriptor systems in [9]. For a NDAS subjected to nonvanishing disturbance, the issue of how to design a controller such that the corresponding closed-loop system is finite-time stable has not been addressed.

In this paper, we will consider the finite-time stabilization problem for a class of NDASs subject to external disturbance. To deal with this problem, motivated by the recently developed disturbance observer based control technique [18, $22,23,39,40]$, a composite control approach is obtained by using the adding a power integrator technique [41] and homogeneous system theory [42]. It is shown that under the proposed composite controller the corresponding closedloop system is finite time stable even in the presence of the nonvanishing external disturbance. The block diagram of the proposed composite control scheme is described by Figure 1. The general design procedure of the composite controller is given according to the following steps. Firstly, when there is no disturbance in NDAS, under some mild conditions, a nominal FTC law is designed recursively by using the adding a power integrator technique. Due to the domination nature of the adopted method, the algebraic variables are not needed to be solved from the algebraic constraints explicitly, and only differential variables are involved in the proposed nominal FTC law. Secondly, to estimate the disturbance, a continuous finite-time disturbance observer (CFTDO) is constructed based on homogeneous systems theory. It is shown that under the proposed CFTDO the disturbance can be estimated precisely in finite time. Finally, based on the proposed nominal FTC law and the estimation of the disturbance, a CFTC is constructed. Rigorous theoretical analysis shows that the proposed CFTC will render the closed-loop system finite-time stable even in the presence

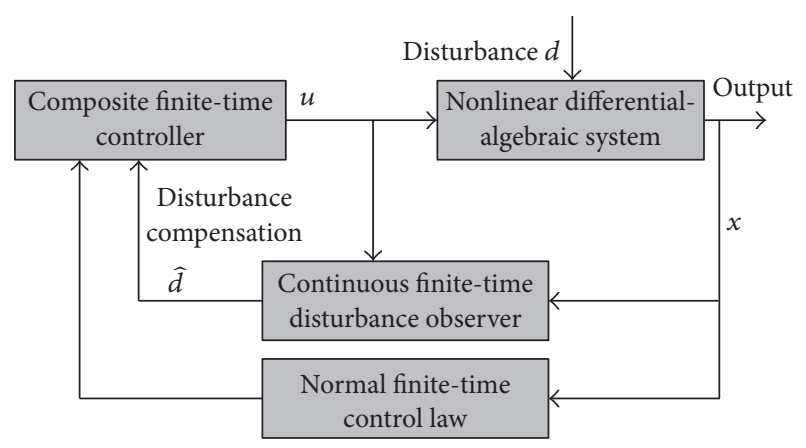

FIGURE 1: The block diagram of the proposed composite control scheme.

of nonvanishing disturbance. With the proposed composite control approach, the disturbance rejection ability of the system is significantly improved without sacrificing the nominal performance recovery property.

The remainder of the paper is organized as follows. In the next section, the problem description is given. Section 3 presents the main result of this paper including the design of nominal FTC law, CFTO, and the CFTC for the NDAS. In Section 4, the effectiveness of the proposed control algorithm is testified by employing a simulation example. Some concluding remarks are included in Section 5. Appendices A and B collect the preliminaries and the proofs of several key propositions, respectively.

\section{Problem Description}

Consider the following NDASs:

$$
\begin{aligned}
\dot{x}_{i} & =x_{i+1}+\phi_{i}(x, z), \quad i=1, \ldots, n-1, \\
\dot{x}_{i} & =u+\phi_{i}(x, z)+d, \\
0 & =\varphi_{i}\left(\underline{x}_{i}, \underline{z}_{i}\right), \quad i=1, \ldots, n,
\end{aligned}
$$

where $x=\left(x_{1}, \ldots, x_{n}\right)^{T} \in \mathbb{R}^{n}$ is a vector of differential variables, $z=\left(z_{1}, \ldots, z_{n}\right)^{T} \in \mathbb{R}^{n}$ is a vector of algebraic variables, $u \in \mathbb{R}$ is the control input, $d \in \mathbb{R}$ is the external disturbance, respectively; $\underline{x}_{i}=\left(x_{i}, \ldots, x_{2}, x_{1}\right)^{T}$ and $\underline{z}_{i}=\left(z_{i}, \ldots, z_{2}, z_{1}\right)^{T}$, $i=1, \ldots, n, \phi_{i}, i=1, \ldots, n$, are continuous nonlinear functions, and nonlinear functions $\varphi_{i} \in \mathbb{C}^{1}, i=1, \ldots, n$. Assume that the origin is an isolated equilibrium point: that is, $\phi_{i}(0,0)=0, \varphi_{i}(0,0)=0, i=1, \ldots, n$.

For NDASs (1a) and (1b), it is supposed that the disturbance $d$ is generated by the following exogenous system:

$$
\begin{aligned}
& \dot{\eta}=A \eta+B \theta, \\
& d=C \eta,
\end{aligned}
$$

where $\eta=\left[\begin{array}{l}\eta_{1} \\ \eta_{2}\end{array}\right]$, the matrices $A=\left[\begin{array}{ll}0 & 1 \\ a_{1} & a_{2}\end{array}\right], B=\left[\begin{array}{l}0 \\ 1\end{array}\right]$, and $C=$ $\left[\begin{array}{ll}1 & 0\end{array}\right]$, and $a_{1}$ and $a_{2}$ are known constants while the constant $\theta$ is not assumed to be known, which means that $d$ may be a type of nonvanishing disturbance. 
Remark 1. In many cases, the disturbance is considered to be generated by a continuous ecosystem [18, 19, 25-27]. It can be verified that, with different parameters $a_{1}, a_{2}$, and $\theta$, (2) can be used to describe a wide class of disturbances, such as constant disturbance [22], ramp disturbance [20], harmonic disturbance [18], and polynomial disturbance [21]. It is well known that the existence of disturbance will bring bad effects to practical engineering system. Therefore, it is no doubt that if the disturbances $d$ can be estimated precisely in finite time, then use its estimation $\widehat{d}$ to compensate it by using proper feedback control. The obtained composite feedback control will enhance the disturbance rejection ability as well as increase control precision of the considered control system.

The control objective is to design a composite controller for NDASs (1a) and (1b) such that the closed-loop system is finite-time stable even in the presence of disturbance.

To solve the finite-time stabilization problem of NDASs (1a) and (1b), the following two assumptions are needed.

Assumption 2. There are two constants $\tau_{1} \in(-1 / n, 0)$ and $\tau_{2} \geq 0$ such that

$$
\begin{aligned}
\left|\phi_{i}\right| & \leq c_{i}\left(\underline{x}_{i}\right)\left(\left|x_{1}\right|^{\left(r_{i}+\tau_{1}\right) / r_{1}}+\cdots+\left|x_{i}\right|^{\left(r_{i}+\tau_{1}\right) / r_{i}}\right. \\
& \left.+\left|z_{1}\right|^{\left(r_{i}+\tau_{1}\right) / m_{1}}+\cdots+\left|z_{i}\right|^{\left(r_{i}+\tau_{1}\right) / m_{i}}\right), \quad i=1, \ldots, n,
\end{aligned}
$$

with

$$
\begin{aligned}
r_{1} & =1, \\
r_{i} & =r_{i-1}+\tau_{1}, \quad i=2, \ldots, n+1, \\
m_{1} & =1, \\
m_{i} & =m_{i-1}+\tau_{2}, \quad i=2, \ldots, n,
\end{aligned}
$$

where $c_{i}\left(\underline{x}_{i}\right) \geq 0$ is a known $\mathbb{C}^{1}$ function.

Remark 3. In the literature, most of the results for NDASs are obtained based on the assumption that the nonlinear functions need to meet smooth conditions [2, 3, 7-10, 15$17,22,25,26]$ or Lipschitz conditions [27, 43]. However, it is observed that, from Assumption 2, the nonlinear terms $f_{i}, i=1, \ldots, n$ in system (1a) may violate these two conditions, which means that (1a) and (1b) may be a non-Lipschitz continuous system [44, 45]. Obviously, system (1a) and (1b) under Assumption 2 can be used to describe a more general class of NDASs.

Assumption 4. There exist a positive constant $\widehat{c}_{2 i}$ and a $\mathbb{C}^{1}$ function $\bar{b}_{i}\left(\underline{x}_{i}\right) \geq 0$ such that

$$
\begin{aligned}
& \frac{\partial g_{i}(\cdot)}{\partial z_{i}} \geq \widehat{c}_{2 i}>0, \\
& \left|g_{i}\left(0, z_{i-1}, \ldots, z_{1}, \underline{x}_{i}\right)\right| \leq \bar{b}_{i}\left(\underline{x}_{i}\right)\left(\left|x_{1}\right|^{m_{i} / r_{1}}+\cdots\right. \\
& \left.\quad+\left|x_{i}\right|^{m_{i} / r_{i}}+\left|z_{1}\right|^{m_{i} / m_{1}}+\cdots+\left|z_{i-1}\right|^{m_{i} / m_{i-1}}\right), \\
& i=1, \ldots, n .
\end{aligned}
$$

For the simplicity, we assume that $\tau_{1}=q_{1} / p_{1}$ and $\tau_{2}=$ $q_{2} / p_{2}$ with $q_{1}$ and $q_{2}$ being even integers and $p_{1}$ and $p_{2}$ being odd integers. Under this assumption, $r_{i}$ and $m_{i}$ will always be odd in both denominator and numerator.

Remark 5. For Assumption 4, the following two points need to be explained.

(i) The condition $\partial \varphi_{i}(\cdot) / \partial z_{i} \neq 0, i=1, \ldots, n$ in Assumption 4 allows us to infer the NDAS behavior to some extent from results on normal systems [2]. In fact, if we define $\varphi(x, z)=\left[\varphi_{1}, \ldots, \varphi_{n}\right]^{T}$, and $\Omega=\left\{(x, z) \in \mathbb{R}^{2 n}: 0=\varphi(x, z)\right\}$, then it follows from Assumption 4 that the Jacobian of $\varphi(x, z)$ with respect to $z$ has full rank on $\Omega$; that is, NDASs (1a) and (1b) are index one, which can guarantee that the NDASs (1a) and (1b) are impulse free and have at least one solution for any consistent initial conditions $(x(0), z(0))$ satisfying $\varphi(x(0), z(0))=0$

(ii) Based on Assumption 4, we have

$$
\begin{aligned}
0= & g_{i}\left(\underline{z}_{i}, \underline{x}_{i}\right)=g_{i}\left(z_{i}, z_{i-1}, \ldots, z_{1}, \underline{x}_{i}\right) \\
= & g_{i}\left(z_{i}, z_{i-1}, \ldots, z_{1}, \underline{x}_{i}\right)-g_{i}\left(0, z_{i-1}, \ldots, z_{1}, \underline{x}_{i}\right) \\
& +g_{i}\left(0, z_{i-1}, \ldots, z_{1}, \underline{x}_{i}\right) \\
= & \int_{0}^{1} \frac{\partial g_{i}\left(\lambda z_{i}, z_{i-1}, \ldots, z_{1}, \underline{x}_{i}\right)}{\partial z_{i}} d \lambda z_{i} \\
& +g_{i}\left(0, z_{i-1}, \ldots, z_{1}, \underline{x}_{i}\right) .
\end{aligned}
$$

It follows from (6) that $\int_{0}^{1}\left(\partial g_{i}\left(\lambda z_{i}, z_{i-1}, \ldots, z_{1}, x_{i}\right) / \partial z_{i}\right) d \lambda z_{i}=$ $-g_{i}\left(0, z_{i-1}, \ldots, z_{1}, \underline{x}_{i}\right)$. According to (5), it can be shown that

$$
\begin{gathered}
\widehat{c}_{2 i}\left|z_{i}\right| \leq\left|\int_{0}^{1} \frac{\partial g_{i}\left(\lambda z_{i}, z_{i-1}, \ldots, z_{1}, \underline{x}_{i}\right)}{\partial z_{i}} d \lambda z_{i}\right| \\
=\left|-g_{i}\left(0, z_{i-1}, \ldots, z_{1}, \underline{x}_{i}\right)\right| \leq \bar{b}_{i}\left(\underline{x}_{i}\right)\left(\left|x_{1}\right|^{m_{i} / r_{1}}+\cdots\right. \\
\left.\quad+\left|x_{i}\right|^{m_{i} / r_{i}}+\left|z_{1}\right|^{m_{i} / m_{1}}+\cdots+\left|z_{i-1}\right|^{m_{i} / m_{i-1}}\right) .
\end{gathered}
$$

This implies that the following inequality holds:

$$
\begin{aligned}
& \left|z_{i}\right| \leq \frac{\check{c}_{2 i}}{\widehat{c}_{2 i}}\left(\left|x_{1}\right|^{m_{i} / r_{1}}+\cdots+\left|x_{i}\right|^{m_{i} / r_{i}}+\left|z_{1}\right|^{m_{i} / m_{1}}+\cdots\right. \\
& \left.\quad+\left|z_{i-1}\right|^{m_{i} / m_{i-1}}\right) \leq b_{i}\left(\underline{x}_{i}\right)\left(\left|x_{1}\right|^{m_{i} / r_{1}}+\cdots+\left|x_{i}\right|^{m_{i} / r_{i}}\right. \\
& \left.\quad+\left|z_{1}\right|^{m_{i} / m_{1}}+\cdots+\left|z_{i-1}\right|^{m_{i} / m_{i-1}}\right)
\end{aligned}
$$

with $b_{i}\left(\underline{x}_{i}\right)=\bar{b}_{i}\left(\underline{x}_{i}\right) / \widehat{c}_{2 i}$. This means that the algebraic variable $z_{i}$ could be bounded by homogeneous-like polynomial.

With the help of Assumption 4, we are able to handle a wide class of NDASs. It is obvious that when NDASs (1a) and (1b) are of index one, by implicit function theorem, there exists a function $h(x)$ so that $z=h(x)$. However, there are many NDASs whose algebraic constraints could represent severe nonlinearities. In this condition, the algebraic variables might not be solved explicitly from the nonlinear algebraic 
constraints, but they could be bounded by homogeneouslike polynomials. For example, consider the following NDAS without disturbance:

$$
\begin{gathered}
\dot{x}=u+\sin (x) z, \\
g(z, x)=5 z+3 \sin z+2 x^{3}=0 .
\end{gathered}
$$

It can be easily proved that $\partial g(z, x) / \partial z \neq 0$. But it is difficult to solve $z$ explicitly from the nonlinear algebraic constrains $g(z, x)$. In fact, according to the nonlinear algebraic $g(z, x)=0$, we have

$$
\begin{aligned}
5|z| & =\left|-3 \sin z-2 x^{3}\right| \leq 3|\sin (z)|+2|x|^{3} \\
& \leq 3|z|+2 x^{2}|x| .
\end{aligned}
$$

It is can be easily deduced from (10) that

$$
|z| \leq b(x)|x|
$$

with $b(x)=x^{2}$, which is in the form of (5).

\section{Composite Controller Design and Stability Analysis}

In this section, we will focus on solving the finite-time stabilization problem of NDASs (1a) and (1b) with the disturbance generated by the exogenous system (2). The detailed design and analysis procedure is divided into three parts, and we will present it step by step.

3.1. Part I: Nominal FTC Law Design for NDASs (1a) and (1b). In this part, we will propose the nominal FTC law design method for NDASs (1a) and (1b) without considering external disturbance. Based on this nominal FTC law, in what follows, a composite controller will be constructed to enhance the disturbance rejection ability and the accuracy of the closedloop system.

Theorem 6. Consider NDASs (1a) and (1b) without external disturbance; if Assumptions 2 and 4 hold, then there exists a FTC law rendering system (1a) and (1b) finite-time stable.

Proof. The proof of this theorem will be carried out in an inductive argument manner which will enable us to construct a $\mathbb{C}^{1}$ Lyapunov function and a $\mathbb{C}^{0}$ virtual control law at each step.

Initial Step. For system (1a) and (1b), choose Lyapunov function $V_{1}\left(x_{1}\right)=\int_{0}^{x_{1}}\left(s^{1 / r_{1}}-0\right)^{2-\tau_{1}-r_{1}} d s$. The time derivative of $V_{1}$ along the trajectory of (1a) and (1b) is

$$
\dot{V}_{1}\left(x_{1}\right)=x_{1}^{\left(2-\tau_{1}-r_{1}\right) / r_{1}}\left(x_{2}+\phi_{1}(x, z, u)\right) \text {. }
$$

By Assumption 4, we have $\left|z_{1}\right|^{\left(r_{1}+\tau_{1}\right) / m_{1}} \leq b_{1}^{\left(r_{1}+\tau_{1}\right) / m_{1}}\left(x_{1}\right)$. $\left|x_{1}\right|^{\left(r_{1}+\tau_{1}\right) / r_{1}}$. It follows from Assumption 2 that $\left|\phi_{1}(x, z)\right| \leq$ $d_{1}\left(x_{1}\right)\left|x_{1}\right|^{\left(r_{1}+\tau_{1}\right) / r_{1}}$ with $d_{1}\left(x_{1}\right)=c_{1}\left(x_{1}\right)\left(1+b_{1}^{\left(r_{1}+\tau_{1}\right) / m_{1}}\left(x_{1}\right)\right)$.
Hence,

$$
\begin{aligned}
& \dot{V}_{1}\left(x_{1}\right) \\
& \quad \leq x_{1}^{\left(2-\tau_{1}-r_{1}\right) / r_{1}}\left(x_{2}-x_{2}^{*}+x_{2}^{*}+d_{1}\left(x_{1}\right) x_{1}^{\left(r_{1}+\tau_{1}\right) / r_{1}}\right) .
\end{aligned}
$$

Clearly, the virtual control law $x_{2}^{*}$ defined by $x_{2}^{*}=-(n+$ $\left.d_{1}\left(x_{1}\right)\right) x_{1}^{r_{2} / r_{1}}:=-\beta_{1}\left(x_{1}\right) x_{1}^{r_{2} / r_{1}}$, where $\beta_{1}\left(x_{1}\right)$ is a smooth, nonnegative function, leads to

$$
\dot{V}_{1}\left(x_{1}\right) \leq-n x_{1}^{2 / r_{1}}+x_{1}^{\left(2-\tau_{1}-r_{1}\right) / r_{1}}\left(x_{2}-x_{2}^{*}\right) .
$$

Inductive Step. Suppose at step $i-1$ that there exist a $\mathbb{C}^{1}$ Lyapunov function $V_{i-1}\left(\underline{x}_{i-1}\right)$, which is positive definite and homogeneous with respect to the dilation $\left(r_{1}, r_{2}, \ldots, r_{i-1}\right)$, and a set of $\mathbb{C}^{0}$ virtual control laws $x_{1}^{*}, \ldots, x_{i}^{*}$, defined by

$$
\begin{aligned}
& x_{1}^{*}=0, \\
& \xi_{1}=x_{1}^{1 / r_{1}}-x_{1}^{* 1 / r_{1}}, \\
& x_{k}^{*}=-\beta_{k-1}\left(\underline{x}_{k-1}\right) \xi_{k-1}^{r_{k}}, \\
& \xi_{k}=x_{k}^{1 / r_{k}}-x_{k}^{* 1 / r_{k}},
\end{aligned}
$$

$$
k=2, \ldots, i
$$

with smooth functions $\beta_{1}(\cdot)>0, \ldots, \beta_{i-1}(\cdot)>0$, such that

$$
\dot{V}_{i-1}\left(\underline{x}_{i-1}\right) \leq-(n-i+2) \sum_{k=1}^{i-1} \xi_{k}^{2}+\xi_{i-1}^{2-\tau_{1}-r_{i-1}}\left(x_{i}-x_{i}^{*}\right) \text {. }
$$

We claim that (16) still holds at step $i$. To prove this claim, we consider the Lyapunov function

$$
V_{i}\left(\underline{x}_{i}\right)=V_{i-1}\left(\underline{x}_{i-1}\right)+W_{i}\left(\underline{x}_{i}\right),
$$

with $W_{i}\left(\underline{x}_{i}\right)=\int_{x_{i}^{*}}^{x_{i}}\left(s^{1 / r_{i}}-x_{i}^{* 1 / r_{i}}\right)^{2-\tau_{1}-r_{i}} d s$. By using a similar method in [45], it can be proved that the Lyapunov function (17) is a $\mathbb{C}^{1}$ positive definite function.

Taking the derivative of the Lyapunov function $V_{i}$ along system (1a) and (1b) yields

$$
\begin{aligned}
\dot{V}_{i}\left(\underline{x}_{i}\right) \leq & -(n-i+2) \sum_{k=1}^{i-1} \xi_{k}^{2}+\xi_{i-1}^{2-\tau_{1}-r_{i-1}}\left(x_{i}-x_{i}^{*}\right) \\
& +\sum_{k=1}^{i-1} \frac{\partial W_{i}}{\partial x_{k}} \dot{x}_{k}+\frac{\partial W_{i}}{\partial x_{i}} \dot{x}_{i} \\
= & -(n-i+2) \sum_{k=1}^{i-1} \xi_{k}^{2}+\xi_{i-1}^{2-\tau_{1}-r_{i-1}}\left(x_{i}-x_{i}^{*}\right) \\
& +\xi_{i}^{2-\tau_{1}-r_{i}}\left(x_{i+1}^{*}+\phi_{i}(\cdot)\right)+\sum_{k=1}^{i-1} \frac{\partial W_{i}}{\partial x_{k}} \dot{x}_{k} \\
& +\xi_{i}^{2-\tau_{1}-r_{i}}\left(x_{i+1}-x_{i+1}^{*}\right)
\end{aligned}
$$

with a virtual control law $x_{i}^{*}$ to be determined later. 
To proceed further, we need to estimate each term of the right hand side of (18). For the second term in (18), based on the fact $r_{i} \leq 1$ and Lemmas A.6 and A.7, it can be shown that there exists a constant $a_{1}>0$ such that

$$
\begin{aligned}
& \xi_{i-1}^{2-\tau_{1}-r_{i-1}}\left(x_{i}-x_{i}^{*}\right) \\
& \quad \leq\left|\xi_{i-1}\right|^{2-\tau_{1}-r_{i-1}}\left|\left(x_{i}^{1 / r_{i}}\right)^{r_{i}}-\left(x_{i}^{*\left(1 / r_{i}\right)}\right)^{r_{i}}\right| \\
& \quad \leq\left|\xi_{i-1}\right|^{2-\tau_{1}-r_{i-1}} 2^{1-r_{i}}\left|x_{i}^{1 / r_{i}}-x_{i}^{*\left(1 / r_{i}\right)}\right|^{r_{i}} \\
& \quad=\left|\xi_{i-1}\right|^{2-\tau_{1}-r_{i-1}} 2^{1-r_{i}}\left|\xi_{i}\right|^{r_{i}} \leq \frac{1}{3} \xi_{i-1}^{2}+a_{1} \xi_{i}^{2} .
\end{aligned}
$$

To estimate the third term and the fourth term of the right hand side of inequality (18), the following propositions are introduced, the proofs of which are included in Appendix B.

Proposition 7. There exists $a \mathbb{C}^{\infty}$ function $a_{2}\left(\underline{x}_{i}\right)>0$ such that

$$
\begin{aligned}
\xi_{i}^{\left(2 \mu-\tau_{1}-r_{i}\right) / \mu} \phi_{i}(\cdot) \leq & \frac{1}{2}\left(\xi_{1}^{2}+\xi_{2}^{2}+\cdots+\xi_{i-2}^{2}\right)+\frac{1}{3} \xi_{i-1}^{2} \\
& +a_{2}\left(\underline{x}_{i}\right) \xi_{i}^{2} .
\end{aligned}
$$

Proposition 8. There exists a smooth function $a_{3}\left(\underline{x}_{i}\right)>0$ such that

$$
\begin{aligned}
\left|\sum_{k=1}^{i-1} \frac{\partial W_{i}}{\partial x_{k}} \dot{x}_{k}\right| \leq & \frac{1}{2}\left(\xi_{1}^{2}+\xi_{2}^{2}+\cdots+\xi_{i-2}^{2}\right)+\frac{1}{3} \xi_{i-1}^{2} \\
& +a_{3}\left(\underline{x}_{i}\right) \xi_{i}^{2}
\end{aligned}
$$

Substituting the estimates (19), (B.3), and (21) into (18) yields

$$
\begin{aligned}
\dot{V}_{i}\left(\underline{x}_{i}\right) & \\
\leq & -(n-i+1) \sum_{k=1}^{i-1} \xi_{k}^{2} \\
& +\xi_{i}^{2-\tau_{1}-r_{i}}\left(x_{i+1}^{*}+\left(a_{1}+a_{2}\left(\underline{x}_{i}\right)+a_{3}\left(\underline{x}_{i}\right)\right) \xi_{i}^{r_{i}+\tau_{1}}\right) \\
& +\xi_{i}^{2-\tau_{1}-r_{i}}\left(x_{i+1}-x_{i+1}^{*}\right) .
\end{aligned}
$$

Clearly, if the virtual control law is chosen as

$$
\begin{aligned}
x_{i+1}^{*} & =-\beta_{i} \xi_{i}^{r_{i}+\tau_{1}} \\
& =-\left(n-i+1+a_{1}+a_{2}\left(\underline{x}_{i}\right)+a_{3}\left(\underline{x}_{i}\right)\right) \xi_{i}^{r_{i}+\tau_{1}},
\end{aligned}
$$

then we have

$$
\dot{V}_{i}\left(\underline{x}_{i}\right) \leq-(n-i+1) \sum_{k=1}^{i} \xi_{k}^{2}+\xi_{i}^{2-\tau_{1}-r_{i}}\left(x_{i+1}-x_{i+1}^{*}\right) .
$$

This completes the inductive proof.

By the inductive argument, it is obvious that (16) still holds as $i=n$. That is, at the last step, we are able to design a $\mathbb{C}^{1}$ Lyapunov function $V_{n}\left(\underline{x}_{n}\right)$, and a $\mathbb{C}^{0}$ virtual control law $x_{n+1}^{*}=-\beta_{n}\left(\underline{x}_{n}\right) \xi_{n}^{r_{n}+\tau_{1}}$ such that

$$
\dot{V}_{n}\left(\underline{x}_{n}\right) \leq-\sum_{k=1}^{n} \xi_{k}^{2}+\xi_{n}^{2-\tau_{1}-r_{n}}\left(u-x_{n+1}^{*}\right) .
$$

By Definition A.3 and Lemma A.4, it can be shown that the homogeneous degree of $V_{n}$ and $\sum_{k=1}^{n} \xi_{k}^{2}$ are $2-\tau_{1}$ and 2 with respect to the delation $\left(r_{1}, \ldots, r_{n}\right)$, respectively. By using Lemma A.4 again, there exists a positive constant $c_{0}$ such that $\sum_{k=1}^{n} \xi_{k}^{2} \geq c_{0} V_{n}^{2 /\left(2-\tau_{1}\right)}$.

Obviously, if we choose

$$
\begin{aligned}
u= & x_{n+1}^{*}=-\beta_{n}\left(\underline{x}_{n}\right) \xi_{n}^{r_{n}+\tau_{1}}=-\beta_{n}\left(\underline{x}_{n}\right)\left(x_{n}^{1 / r_{n}}\right. \\
& +\beta_{n-1}\left(\underline{x}_{n-1}\right)\left(x_{n-1}^{1 / r_{n-1}}+\cdots\right. \\
& \left.\left.+\beta_{2}\left(\underline{x}_{2}\right)\left(x_{2}^{1 / r_{2}}+\beta_{1}\left(x_{1}\right) x_{1}\right) \cdots\right)\right)^{r_{n}+\tau_{1}},
\end{aligned}
$$

then by using Lemma A.6, we have

$$
\dot{V}_{n}\left(\underline{x}_{n}\right) \leq-\sum_{k=1}^{n} \xi_{k}^{2} \leq-c_{0} V_{n}^{2 /\left(2-\tau_{1}\right)} .
$$

Note that $\tau_{1}<0$; thus, $2 /\left(2-\tau_{1}\right) \in(0,1)$. By Lemma A.2, the states of subsystem (1a) will converge to the origin in finite time; that is, there exists a time constant $T_{1}$ such that $x_{i}(t) \equiv$ $0, i=1, \ldots, n, \forall t \geq T_{1}$.

Based on this and Assumption 4, let $t \geq T_{1}$; it follows from $\left|z_{1}\right| \leq b_{1}\left(x_{1}\right)\left|x_{1}\right|^{m_{1} / r_{1}}$ that $z_{1} \equiv 0$ as $t \geq T_{1}$. And then based on the fact $x_{1} \equiv 0, x_{2} \equiv 0, z_{1} \equiv 0$ as $t \geq T_{1}$, and $\left|z_{2}\right| \leq b_{2}\left(\underline{x}_{2}\right)\left(\left|x_{1}\right|^{m_{2} / r_{1}}+\left|x_{2}\right|^{m_{2} / r_{2}}+\left|z_{1}\right|^{m_{2} / m_{1}}\right)$, we have $z_{2} \equiv 0$ as $t \geq T_{1}$. Proceeding in the same line, it can be shown that $z_{1} \equiv 0, \ldots, z_{n} \equiv 0, \forall t \geq T_{1}$ step by step.

Therefore, it can be concluded that the closed-loop system (1a) and (1b) and (26) are finite-time stable.

Remark 9. It is generally known that the selection of Lyapunov function plays a central role in the control design procedure. For NDAS in an alternative expression

$$
E \dot{\bar{x}}=f(\bar{x}) \text {, }
$$

where $\bar{x} \in \mathbb{R}^{n}, f: \mathbb{R}^{n} \rightarrow \mathbb{R}^{n}$ is smooth enough and $f(0)=0$, and $E \in \mathbb{R}^{n \times n}$ is a singular matrix with $\operatorname{rank}(E)=$ $r<n$. According to [10,44], if NDAS (28) is index one, then there exist two nonsingular matrices $M, N \in \mathbb{R}^{n \times n}$ such that $M E N=\left[\begin{array}{cc}I_{r} & 0 \\ 0 & 0\end{array}\right]$. That is, if we let $x=N \bar{x}$, and $M f(N x)=\left[\begin{array}{l}f_{1}\left(x_{1}, x_{2}\right) \\ f_{2}\left(x_{1}, x_{2}\right)\end{array}\right]$, then system (28) is equivalent to the system described by $\dot{x}_{1}=f_{1}\left(x_{1}, x_{2}\right), 0=f_{2}\left(x_{1}, x_{2}\right)$, with $x_{1} \in \mathbb{R}^{r}$ and $x_{2} \in \mathbb{R}^{n-r}$. However, due to the inherent mixed differential-algebraic structure for DASs, the selection of Lyapunov function and the calculation of the derivative of the Lyapunov function along the trajectory of the systems are more difficult than those of the normal systems [22]. It is obvious that the finite-time stabilization problem of NDAS (la) and (lb) is not a trivial issue. To study the finitetime stabilization problem of system (1a) and (1b) without disturbance, our motivation is twofold. 
(i) From the energy perspective, the algebraic constraints can be naturally regarded as the generalized internal energy balance, so the algebraic constraints do not affect energy balance between the system and external world [24]. And in [10], it was pointed out that for the index one descriptor system (28) only the part where $E \bar{x} \neq 0$ contributes to the energy function.

(ii) To stabilize a linear singular system, it is sufficient to finish this task by applying slow feedback [4].

Based on these two points and the merits of the adding a power integrator technique, we have proved that it is feasible to design Lyapunov function and FTC control law for NDAS (la) and (lb) by only using the differential variables.

In the absence of disturbance, Theorem 6 provides a finite-time stabilization approach for NDASs (1a) and (1b). However, in the presence of external disturbance, the proposed control approach cannot guarantee that the states of the system converge to the origin accurately in finite time. Inspired by the recently developed disturbance observer based control technique $[18,20,22,27]$, we will design a continuous nonlinear disturbance observer such that the disturbance can be estimated in finite time, and then combining the proposed FTC law and the disturbance estimation a CFTC will be given in the next part.

3.2. Part II: CFTDO Design. In this part, we will concentrate on the CFTDO for system (1a) and (1b) by using homogeneous system theory.

Combining (1a) and (2), we define

$$
\begin{aligned}
& y_{1}=x_{n}, \\
& y_{2}=\eta_{1}-a_{2} y_{1}, \\
& y_{3}=\eta_{2}-a_{2} \eta_{1}-a_{1} y_{1}, \\
& y_{4}=\theta .
\end{aligned}
$$

Based on (1a), (2), and (29), we get the derivatives of $y_{j}, j=1, \ldots, 4$ that

$$
\begin{aligned}
& \dot{y}_{1}=y_{2}+f_{1}, \\
& \dot{y}_{2}=y_{3}+f_{2}, \\
& \dot{y}_{3}=y_{4}+f_{3}, \\
& \dot{y}_{4}=0,
\end{aligned}
$$

where the nonlinear functions

$$
\begin{aligned}
& f_{1}=a_{2} y_{1}+\phi_{n}(x, z)+u, \\
& f_{2}=a_{1} y_{1}-a_{2}\left(\phi_{n}(x, z)+u\right), \\
& f_{3}=-a_{1}\left(\phi_{n}(x, z)+u\right) .
\end{aligned}
$$

As $a_{1}, a_{2}$ are known constants, and $\phi_{n}(x, z)$ are known nonlinear functions, it is obvious that $f_{i}, i=1,2,3$ are known nonlinear terms.

Therefore, the CFTDO design problem of system (1a) and (1b) is converted to the finite-time state observer for system (30).
Lemma 10. For system (30), there exists a states observer in the following form:

$$
\begin{aligned}
& \dot{\hat{y}}_{1}=\widehat{y}_{2}+f_{1}+k_{1}\left(y_{1}-\widehat{y}_{1}\right)^{\gamma_{2} / \gamma_{1}}, \\
& \dot{\hat{y}}_{2}=\widehat{y}_{3}+f_{2}+k_{2}\left(y_{1}-\widehat{y}_{1}\right)^{\gamma_{3} / \gamma_{1}}, \\
& \dot{\hat{y}}_{3}=\widehat{y}_{4}+f_{3}+k_{3}\left(y_{1}-\widehat{y}_{1}\right)^{\gamma_{4} / \gamma_{1}}, \\
& \dot{\hat{y}}_{4}=k_{4}\left(y_{1}-\widehat{y}_{1}\right)^{\gamma_{5} / \gamma_{1}},
\end{aligned}
$$

where $\gamma_{1}=1, \gamma_{i}=\gamma_{1}+(i-1) \tau_{3}, i=2,3,4,5, \tau_{3} \in(-1 / 4,0)$, and $k_{i}, i=1,2,3,4$ are appropriate positive constants, such that the states of system (30) can be estimated in finite time.

Proof. Let $e_{i}=y_{i}-\widehat{y}_{i}, i=1,2,3,4$; then based on system (30) and (32) the observe error system is obtained:

$$
\begin{aligned}
& \dot{e}_{1}=e_{2}-k_{1} e_{1}^{\gamma_{2} / \gamma_{1}}, \\
& \dot{e}_{2}=e_{3}-k_{2} e_{1}^{\gamma_{3} / \gamma_{1}}, \\
& \dot{e}_{3}=e_{4}-k_{3} e_{1}^{\gamma_{4} / \gamma_{1}}, \\
& \dot{e}_{4}=-k_{4} e_{1}^{\gamma_{5} / \gamma_{1}} .
\end{aligned}
$$

According to Theorem 3.1 in [37], if we choose appropriate constants $k_{i}>0, i=1,2,3,4$; then system (33) is globally finite-time stable. Thus, there exists a constant $T_{2}>0$ such that $\widehat{y}_{i} \equiv y_{i}, \quad i=2,3,4$, as $t \geq T_{2}$.

According to (29) and (2), it is can be shown that

$$
\begin{aligned}
& \widehat{d}=a_{2} y_{1}+\widehat{y}_{2}=\eta_{1}, \\
& \hat{\dot{d}}=\widehat{y}_{3}+a_{2} \eta_{1}+a_{1} y_{1}=\eta_{2},
\end{aligned}
$$

$$
\forall t \geq T_{2}
$$

That is, both the disturbance $d$ and it is derivative $\dot{d}$ can be estimated in finite time.

Remark 11. To estimate disturbances, many available disturbance observer design methods have been proposed in the literature; for example, see $[18-20,23,27]$ and the reference therein. It should be pointed out that most of the existing disturbance observer design approaches can achieve asymptotic estimates of the disturbances only. To enhance the disturbance rejection ability and control accuracy of the system, it is necessary to develop the design method of finite time disturbance observer, such that the disturbance can be estimated in finite time. Under the assumption that the disturbance is high-order differentiable and bounded, the work [32] proposed a noncontinuous finite-time disturbance observer design method by using high-order sliding mode control technique. Different from [32], Lemma 10 proposes a CFTDO design method under the assumption that disturbance is generated by an exogenous system.

With the help of Theorem 6 and Lemma 10, we are able to propose a finite-time stabilization approach for NDASs (1a) and (1b) subject to disturbance. 
3.3. Part III: CFTC Design for NDASs (1a) and (1b). In this part, we will propose a CFTC for NDASs (1a) and (1b) with disturbance, and rigorous stability analysis will be given to guarantee the finite-time stability of the corresponding closed-loop system.

Theorem 12. Consider NDASs (1a) and (1b) subject to the disturbance generated by the exogenous system (2); if Assumptions 2 and 4 hold, then under the controller

$$
\begin{aligned}
u= & -\beta_{n}\left(\underline{x}_{n}\right)\left(x_{n}^{1 / r_{n}}+\beta_{n-1}\left(\underline{x}_{n-1}\right)\left(x_{n-1}^{1 / r_{n-1}}+\cdots\right.\right. \\
& \left.\left.+\beta_{2}\left(\underline{x}_{2}\right)\left(x_{2}^{1 / r_{2}}+\beta_{1}\left(x_{1}\right) x_{1}\right) \cdots\right)\right)^{r_{n}+\tau_{1}}-\widehat{d}
\end{aligned}
$$

the closed-loop system composed of (1a) and (1b) and (35) is finite-time stable.

Proof. For NDASs (1a) and (1b) with disturbance, we choose the same Lyapunov function $V_{n}\left(\underline{x}_{n}\right)$ presented above. In the same line as Theorem 6 , we have

$$
\dot{V}_{n}\left(\underline{x}_{n}\right) \leq-\sum_{k=1}^{n} \xi_{k}^{2}+\xi_{n}^{2-\tau_{1}-r_{n}}\left(u+d-x_{n+1}^{*}\right) .
$$

By Definition A.3 and Lemma A.4, it can be shown that the homogeneous degree of $V_{n}$ and $\sum_{k=1}^{n} \xi_{k}^{2}$ are $2-\tau_{1}$ and 2 with respect to the delation $\left(r_{1}, \ldots, r_{n}\right)$, respectively. And according to Lemma A.4, there exists a positive constant $c_{0}$ such that $\sum_{k=1}^{n} \xi_{k}^{2} \geq c_{0} V_{n}^{2 /\left(2-\tau_{1}\right)}$.

Substituting (35) into inequality (36) and using Lemma A.6 yield

$$
\begin{aligned}
\dot{V}_{n}\left(\underline{x}_{n}\right) \leq & -\sum_{k=1}^{n} \xi_{k}^{2}+\xi_{n}^{2-\tau_{1}-r_{n}}(d-\hat{d}) \\
\leq & -\sum_{k=1}^{n} \xi_{k}^{2}+\frac{2-\tau_{1}-r_{n}}{2} \xi_{n}^{2} \\
& +\frac{\tau_{1}+r_{n}}{2}|d-\widehat{d}|^{2 /\left(\tau_{1}+r_{n}\right)} \\
\leq & -\frac{\tau_{1}+r_{n}}{2} \sum_{k=1}^{n} \xi_{k}^{2}+\frac{\tau_{1}+r_{n}}{2}|d-\widehat{d}|^{2 /\left(\tau_{1}+r_{n}\right)} \\
\leq & -\frac{\tau_{1}+r_{n}}{2} c_{0} V_{n}^{2 /\left(2-\tau_{1}\right)} \\
& +\frac{\tau_{1}+r_{n}}{2}|d-\hat{d}|^{2 /\left(\tau_{1}+r_{n}\right)} .
\end{aligned}
$$

By the analysis and design procedure of the CFTDO, it is easy to know that the observe error system (33) is global finite-time stable. Thus, the states $e_{i}, i=1,2,3,4$ are global bounded. Based on this, and (29) and (34), it is obvious that $d-\widehat{d}$ is global bounded and $d-\widehat{d} \equiv 0, \forall t>T_{2}$. Then, by the definition of FTISS-Lyapunov function in [34], one knows that $V_{n}$ is a FTISS-Lyapunov function of the closedloop system combined by (1a) and (35). By Lemma A.5, it is obvious that the states of subsystem (1a) will converge to the origin in finite time; that is, there exists a time constant $T_{3} \geq T_{2}$ such that $x_{i}(t) \equiv 0, i=1, \ldots, n, \forall t \geq T_{3}$.
By using the similar method as that in Theorem 6 , it can be shown that $z_{1} \equiv 0, \ldots, z_{n} \equiv 0, \forall t \geq T_{3}$ one by one. Therefore, it can be concluded that the NDAS (1) can be stabilized by the composite controller (35) in a finite time.

In order to facilitate the analysis and design, we assume that every subsystem of differential system (1a) has a corresponding algebraic constraint equation for NDASs (1a) and (1b). Note that, for some practical NDASs, parts of the subsystems of differential system (1a) are allowed to not have algebraic constraint equations. Without loss of generality, we assume that the first $n-m$ subsystems of differential system (1a) have no corresponding algebraic constraint equations; then (1b) becomes

$$
0=\varphi_{i}\left(\underline{x}_{n-m+i}, \underline{z}_{i}\right), \quad i=1, \ldots, m .
$$

To state the main result of the finite-time stabilization problems of NDAS (1) when the algebraic system (1b) is in the form of (38), the following two Assumptions which can be seen as modified versions of Assumptions 2 and 4, respectively, are needed.

Assumption $2^{*}$. There are constants $\tau_{1} \in(-1 / n, 0)$ and $\tau_{2} \geq 0$ such that

$$
\begin{array}{r}
\left|\phi_{i}\right| \leq c_{i}\left(\underline{x}_{i}\right)\left(\left|x_{1}\right|^{\left(r_{i}+\tau_{1}\right) / r_{1}}+\cdots+\left|x_{i}\right|^{\left(r_{i}+\tau_{1}\right) / r_{i}}\right), \\
i=1, \ldots, n-m, \\
\left|\phi_{n-m+j}\right| \leq c_{n-m+j}\left(\underline{x}_{n-m+j}\right)\left(\sum_{i=1}^{n-m}\left|x_{i}\right|^{\left(r_{n-m+j}+\tau_{1}\right) / r_{i}}\right. \\
\left.+\sum_{k=1}^{j}\left(\left|x_{n-m+k}\right|^{\left(r_{n-m+j}+\tau_{1}\right) / r_{n-m+k}}+\left|z_{k}\right|^{\left(r_{n-m+j}+\tau_{1}\right) / m_{k}}\right)\right), \\
j=1, \ldots, m,
\end{array}
$$

where $r_{i}$ and $m_{i}$ are defined in Assumption 2, and $c_{i}\left(\underline{x}_{i}\right) \geq 0$ is a known $\mathbb{C}^{1}$ function.

Assumption $4^{*}$. There exist a positive constant $\widehat{c}_{2 i}$ and a $\mathbb{C}^{1}$ function $\bar{b}_{i}\left(\underline{x}_{n-m+i}\right) \geq 0$ such that $\partial g_{i}(\cdot) / \partial z_{i} \geq \widehat{c}_{2 i}>0$ and

$$
\begin{aligned}
& \left|g_{i}\left(0, z_{i-1}, \ldots, z_{1}, \underline{x}_{i}\right)\right| \leq \bar{b}_{i}\left(\underline{x}_{n-m+i}\right)\left(\left|x_{1}\right|^{m_{i} / r_{1}}+\cdots\right. \\
& \quad+\left|x_{n-m}\right|^{m_{i} / r_{n-m}}+\cdots+\left|x_{n-m+i}\right|^{m_{i} / r_{n-m+i}}+\left|z_{1}\right|^{m_{i} / m_{1}} \\
& \left.\quad+\cdots+\left|z_{i-1}\right|^{m_{i} / m_{i-1}}\right), \quad i=1, \ldots, m .
\end{aligned}
$$

Similar to the analysis of Remark 5, it is easy to show that the following conclusion holds: if Assumption $4^{*}$ holds, then there exists a $\mathbb{C}^{1}$ function $b_{i}\left(\underline{x}_{n-m+i}\right)$ such that

$$
\begin{array}{r}
\left|z_{i}\right| \leq b_{i}\left(\underline{x}_{n-m+i}\right)\left(\left|x_{1}\right|^{m_{i} / r_{1}}+\cdots+\left|x_{n-m}\right|^{m_{i} / r_{n-m}}+\cdots\right. \\
\left.+\left|x_{n-m+i}\right|^{m_{i} / r_{n-m+i}}+\left|z_{1}\right|^{m_{i} / m_{1}}+\cdots+\left|z_{i-1}\right|^{m_{i} / m_{i-1}}\right), \\
i=1, \ldots, m .
\end{array}
$$


Based on Assumptions $2^{*}$ and $4^{*}$, in the same lines of the proof of Theorems 6 and 12, the following results can be obtained immediately.

Corollary 13. Consider NDASs (1a) and (1b) without external disturbance; if Assumptions $2^{*}$ and $4^{*}$ hold, then under the feedback control law (26) the corresponding closed-loop system is finite-time stable.

Corollary 14. Consider NDASs (1a) and (1b) subject to the disturbance generated by the exogenous system (2); if Assumptions $2^{*}$ and $4^{*}$ hold, then the composite controller (35) will stabilize system (1) in finite time.

Remark 15. For simplicity, so far we have assumed that $\tau_{i}=$ $-p_{i} / q_{i}, i=1,2$, where $p_{i}$ and $q_{i}$ represent even and odd integers, respectively, such that $r_{i}, m_{i}, i=1, \ldots, n+1$ will be odd in both denominator and numerator. In the case when $\tau_{i}=-p_{i} / q_{i}, i=1,2$, where $p_{i}$ and $q_{i}$ are arbitrary real positive numbers, inspired by $[38,46]$, we define the function $[s]^{r}=\operatorname{sign}(s)|s|^{r}$ to preserve the sign of its variable in the homogeneous finite-time disturbance observer and control law, such that it is still possible to get the same result.

Remark 16. When there are no nonlinear algebraic constraints, the NDASs in (1a) and (1b) reduce to the following normal nonlinear system with disturbance:

$$
\begin{aligned}
& \dot{x}_{i}=x_{i+1}+\phi_{i}(x), \quad i=1, \ldots, n-1, \\
& \dot{x}_{i}=u+\phi_{i}(x)+d
\end{aligned}
$$

In the absence of disturbance, the finite-time stabilization problem of system (42) has been considered in $[33,46]$ under different assumptions on nonlinear terms. In this regard, the obtained results in this paper can be viewed as an extension of existing results on normal nonlinear systems to NDASs. Note that, in the presence of disturbance, the proposed stabilization methods $[33,46]$ can only ensure that the states of the closed-loop system converge to a neighborhood of the origin. However, the obtained composite control method in this paper can effectively enhance the disturbance rejection ability and control accuracy of the system.

Remark 17 (nominal performance recovery ability). In the absence of disturbance, it is derived from the observer error system (33) that $y_{2}=y_{3}=y_{4}=0$ and $e_{i}=0, i=1,2$, 3,4 if the initial values of the observer states are selected as $y_{1}(0)=x_{n}(0)$ and $y_{2}(0)=y_{3}(0)=y_{4}(0)=0$. In this condition, the proposed composite controller (35) reduces to the nominal FTC law (26), which demonstrates that the proposed approach does not sacrifice the nominal performance.

\section{Simulations}

In this section, we will use a numerical example to illustrate the validity of the proposed composite feedback stabilization approach.
Example 18. Consider the following inherent NDAS:

$$
\begin{aligned}
& \dot{x}_{1}=x_{2}+0.2 \sin \left(x_{1}\right), \\
& \dot{x}_{2}=u+0.5 \sin \left(x_{2}\right) z_{1}^{1 / 3}+d(t), \\
& 0=3 z_{1}+z_{1}^{3}-5 x_{1},
\end{aligned}
$$

where the disturbance is generated by exogenous system (2) with $a_{1}=-4, a_{2}=0, \theta=0$, and $d(t)=\eta_{1}=0.8 \sin (2 t)$.

First of all, it is worth mentioning that NDAS (43) is a nonsmooth system because the system contains a nonlinear term $\phi_{2}(x, z)=0.5 \sin \left(x_{2}\right) z_{1}^{1 / 3}$. Obviously, the stabilization method proposed by [27] does not apply to system (43) because the nonlinear term $\phi_{2}(x, z)$ does not meet Lipschitz condition. The recent work [9] proposed a finitetime stabilization for nonsmooth nonlinear Hamiltonian descriptor system. However, the approach proposed by [9] does not apply to system (43) too. How to design a finitetime controller for system (43), there are no existing results to utilise.

However, if we choose $r_{1}=1, m_{1}=1, \tau_{1}=-2 / 9$, and $\tau_{2}=0$, then $r_{2}=7 / 9, r_{3}=5 / 9$. It is easy to show that $\left|\phi_{1}\right|=$ $\left|0.2 \sin \left(x_{1}\right)\right| \leq 0.2\left|x_{1}\right|^{7 / 9}$. According to the nonlinear algebraic constraint $\varphi_{1}$, we have $\partial \varphi_{1}\left(z_{1}, x_{1}\right) / \partial z_{1}=3+3 z_{1}^{2} \neq 0$ and $\varphi_{1}\left(0, x_{1}\right)=-5 x_{1}$. Thus, $\left|z_{1}\right| \leq 5\left|x_{1}\right| /\left(3+z_{1}^{2}\right) \leq(5 / 3)\left|x_{1}\right|$. Furthermore, by using Lemma A.6, it can be easily derived that $\phi_{2}=0.5 \sin \left(x_{2}\right) z_{1}^{1 / 3} \leq 0.5\left|x_{2}\right|^{2 / 7}\left|z_{1}\right|^{3 / 9} \leq(1 / 10)\left(2\left|x_{2}\right|^{5 / 7}+\right.$ $\left.3\left|(5 / 3) z_{1}\right|^{5 / 9}\right) \leq(1 / 2)\left(\left|x_{2}\right|^{5 / 7}+\left|z_{1}\right|^{5 / 9}\right)$. Therefore, Assumptions $2^{*}$ and $4^{*}$ are satisfied. In the absence of external disturbance, according to Corollary 13, the NDAS (43) can be stabilized by the following FTC law:

$$
u=-\beta_{2}\left(\widehat{x}_{2}^{9 / 7}+\beta_{1} x_{1}\right)^{5 / 9}
$$

with appropriate gains $\beta_{1}$ and $\beta_{2}$.

In the presence of disturbance, according to Lemma 10 and Corollary 14, the following CFTC is designed for system (43):

$$
u_{c}=-\beta_{2}\left(\widehat{x}_{2}^{9 / 7}+\beta_{1} x_{1}\right)^{5 / 9}-\widehat{d}
$$

where $\widehat{d}=\widehat{y}_{2}$ is the estimation of the external disturbance generated by the CFTDO

$$
\begin{aligned}
& \dot{\hat{y}}_{1}=\widehat{y}_{2}+\left(0.5 \sin \left(x_{2}\right) z_{1}^{1 / 3}+u_{c}\right)+k_{1}\left(x_{n}-\widehat{y}_{1}\right)^{\gamma_{2} / \gamma_{1}} \\
& \dot{\hat{y}}_{2}=\widehat{y}_{3}-4 x_{n}+k_{2}\left(x_{n}-\widehat{y}_{1}\right)^{\gamma_{3} / \gamma_{1}} \\
& \dot{\hat{y}}_{3}=4\left(0.5 \sin \left(x_{2}\right) z_{1}^{1 / 3}+u_{c}\right)+k_{3}\left(x_{n}-\widehat{y}_{1}\right)^{\gamma_{4} / \gamma_{1}},
\end{aligned}
$$

where $k_{1}, k_{2}$, and $k_{3}$ are appropriate positive constants and $\gamma_{1}=1, \gamma_{i}=\gamma_{1}+(i-1) \tau_{3}, \quad i=2,3,4$ with $\tau_{3} \in(-1 / 3,0)$.

To evaluate the effectiveness of the proposed control approach, FTC (44) and CFTC (45) for system (42) with disturbance are employed in the simulation for the purpose of comparison studies. The control parameters of the two control methods are listed in Table 1 . The initial values of system 


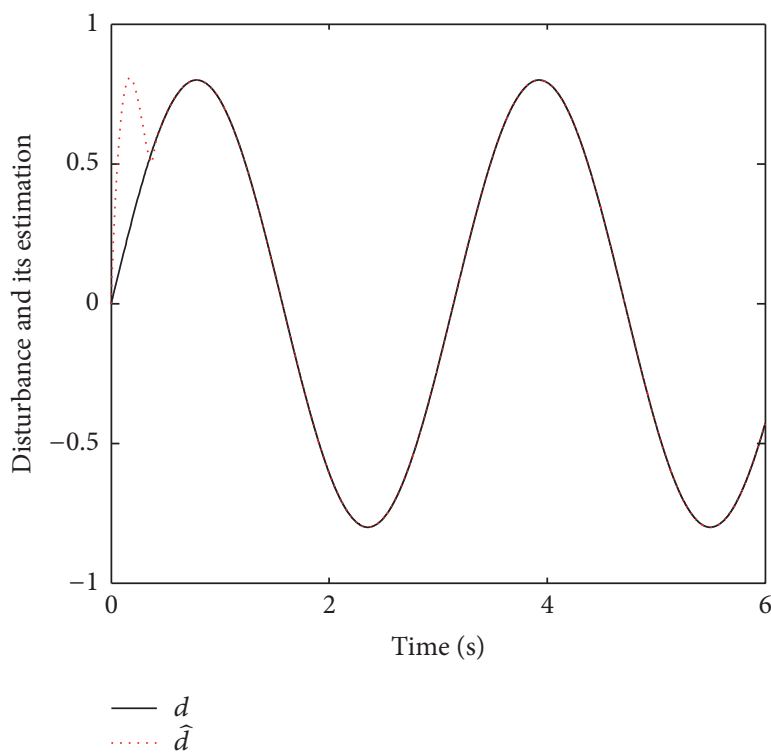

(a)

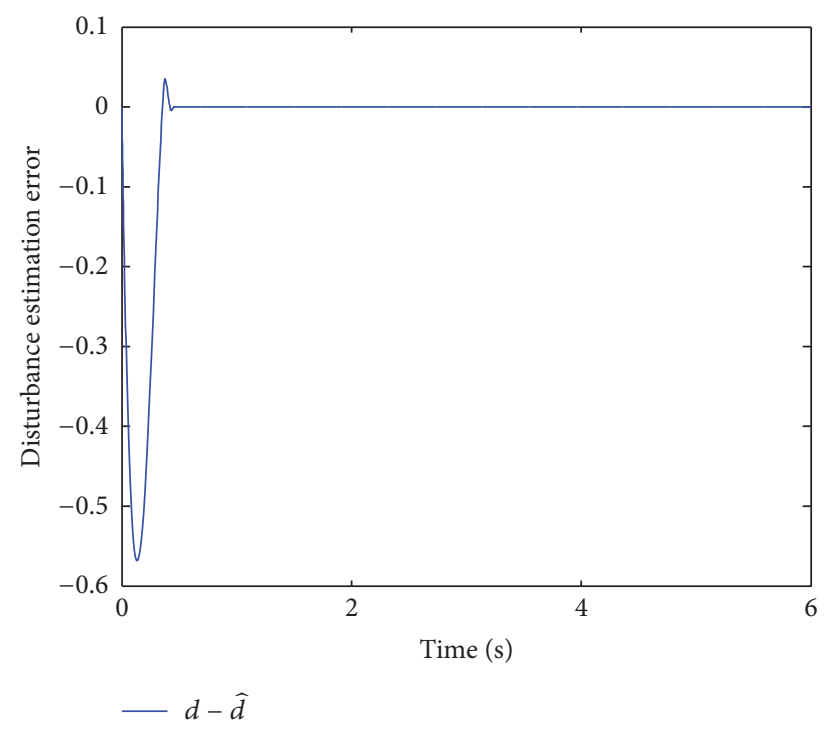

(b)

FIGURE 2: Response curves of disturbance and its estimation (a) and estimation error (b).

TABLE 1: Control parameters for the numerical Example (43).

\begin{tabular}{lc}
\hline Controllers & Parameters \\
\hline FTC: $u$ & $\beta_{1}=2, \beta_{2}=7$ \\
CFTC: $u_{c}$ & $\beta_{1}=2, \beta_{2}=7, k_{1}=10, k_{2}=30, k_{3}=60, \tau_{3}=-2 / 9$ \\
\hline
\end{tabular}

(43) and observer (46) are chosen as $\left(x_{1}(0), x_{2}(0), z_{1}(0)\right)=$ $(2.8,-3,2)$ and $\left(\hat{y}_{1}(0), \hat{y}_{2}(0), \hat{y}_{3}(0)\right)=(-3,0,0)$, respectively. The simulation results are shown in Figures 2 and 3.

It is observed from Figure 2 that the external disturbance can be estimated quickly by the proposed CFTDO (46). The states responses of the NDAS (43) under the two different controls (44) and (45) are shown in Figures 3(a)-3(c), which demonstrate that the FTC law (44) fails to stabilize NDAS (43), while the composite controller (45) can make the states of NDAS (43) converge to the origin precisely. The control signals of the two different controls (44) and (45) are given in Figure 3(d).

Example 19. Consider nonlinear circuit system displayed in Figure 4 , where a dc source with voltage $\mu$ is connected in series to a linear resistor, a linear inductor, and a nonlinear capacitor with a $q-v$ characteristic $q=z(v)=\left(v-v_{0}\right)+(v-$ $\left.v_{0}\right)^{3}+q_{0}$. Similar nonlinear capacitors are considered in $[1,22]$.

This circuit may be easily shown to admit the charge-flux description

$$
\begin{aligned}
\dot{q} & =\frac{\phi}{\mathbf{L}}, \\
\dot{\phi} & =\mu-\frac{\phi \mathbf{R}}{\mathbf{L}}-v, \\
0 & =\left(v-v_{0}\right)^{3}+\left(v-v_{0}\right)-\left(q-q_{0}\right),
\end{aligned}
$$

where $\phi$ is the magnetic flux in the inductor.
Define $x=\left[x_{1}, x_{2}\right]^{T}=\left[q-q_{0}, \phi\right]^{T}, z_{1}=v-v_{0}$, and $u=\mu-v_{0}$. Assume that the system is subject to matched disturbance $d(t)$ which is generated by exogenous system (2) with $a_{1}=-1, a_{2}=0$, and let $\mathbf{L}=1, \mathbf{R}=1.5, v_{0}=1$, and $q_{0}=0.5$; then system (47) can be represented by the following system:

$$
\begin{aligned}
\dot{x}_{1} & =x_{2}, \\
\dot{x}_{2} & =u-1.5 x_{2}-z_{1}+d(t), \\
0 & =z_{1}+z_{1}^{3}-x_{1} .
\end{aligned}
$$

However, if we choose $r_{1}=1, m_{1}=1$, and $\tau_{1}=-2 / 7$; then $r_{2}=5 / 7, r_{3}=3 / 7$. According to the nonlinear algebraic constraint $\varphi_{1}$, we have $\partial \varphi_{1}\left(z_{1}, x_{1}\right) / \partial z_{1}=1+3 z_{1}^{2} \neq 0$ and $\varphi_{1}\left(0, x_{1}\right)=x_{1}$. Thus, $\left|z_{1}\right| \leq\left|x_{1}\right| /\left(1+3 z_{1}^{2}\right) \leq\left|x_{1}\right|$. Furthermore, it is easy to show that $\left|\phi_{2}\right|=\left|-1.5 x_{2}-z_{1}\right| \leq$ $b_{2}\left(x_{1}, x_{2}\right)\left(\left|x_{1}\right|^{3 / 7}+\left|x_{2}\right|^{3 / 5}\right)$ with $b_{2}\left(x_{1}, x_{2}\right)=\left|x_{1}\right|^{4 / 7}+2\left|x_{2}\right|^{2 / 5}$. Therefore, Assumptions $2^{*}$ and $4^{*}$ are satisfied. In the absence of external disturbance, according to Corollary 13, the NDAS (43) can be stabilized by the following FTC law:

$$
u=-\left(10+b_{2}\left(x_{1}, x_{2}\right)\right)\left(\widehat{x}_{2}^{7 / 5}+2 x_{1}\right)^{3 / 7}-\widehat{d},
$$

where $\widehat{d}=\hat{y}_{2}$ is the estimation of the external disturbance generated by the CFTDO

$$
\begin{aligned}
& \dot{\hat{y}}_{1}=\hat{y}_{2}+\left(u-1.5 x_{2}-z_{1}\right)+k_{1}\left(x_{2}-\hat{y}_{1}\right)^{\gamma_{2} / \gamma_{1}}, \\
& \dot{\hat{y}}_{2}=\widehat{y}_{3}-x_{2}+k_{2}\left(x_{2}-\widehat{y}_{1}\right)^{\gamma_{3} / \gamma_{1}}, \\
& \dot{\hat{y}}_{3}=\left(u-1.5 x_{2}-z_{1}\right)+k_{3}\left(x_{2}-\widehat{y}_{1}\right)^{\gamma_{4} / \gamma_{1}}, \\
& \dot{\hat{y}}_{4}=k_{4}\left(x_{2}-\hat{y}_{1}\right)^{\gamma_{5} / \gamma_{1}}
\end{aligned}
$$




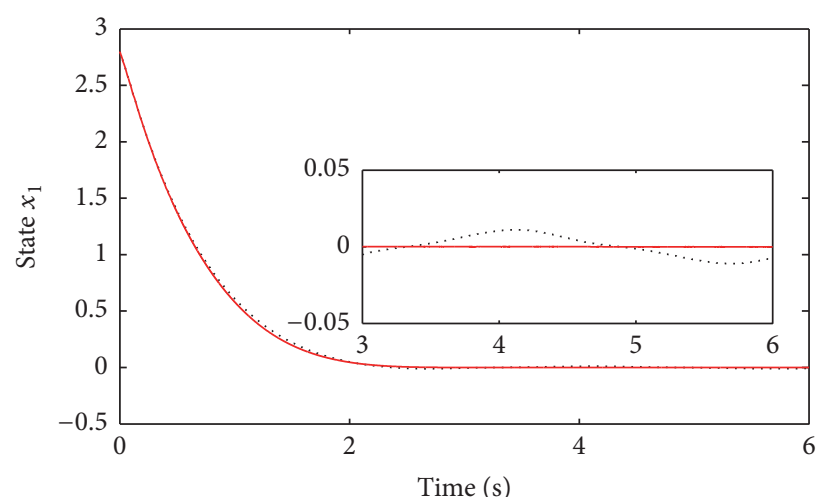

… FTC

- CFTC

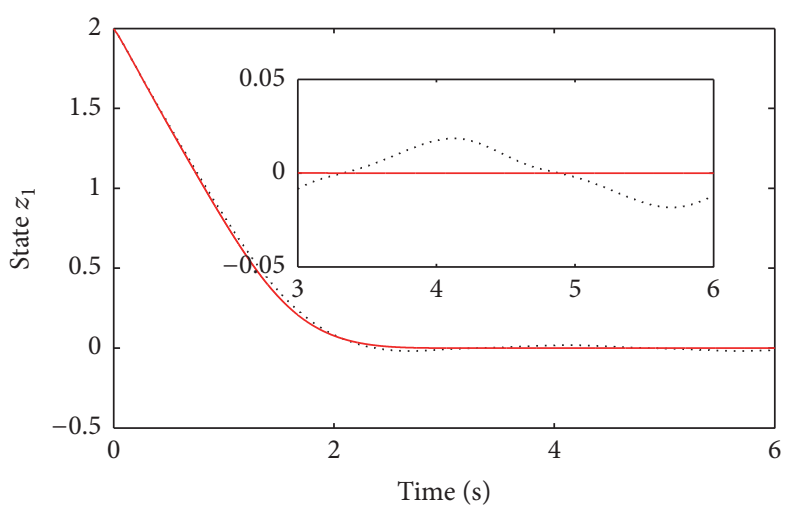

… FTC

- CFTC

(c)

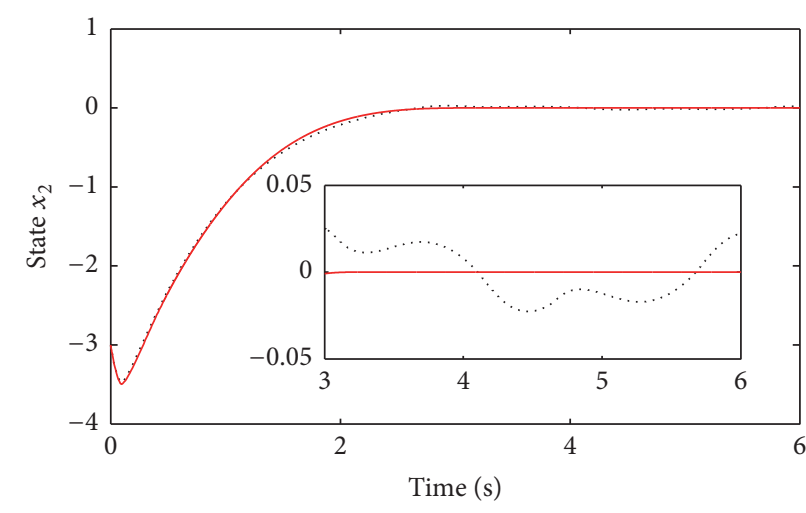

… FTC

- CFTC

(b)

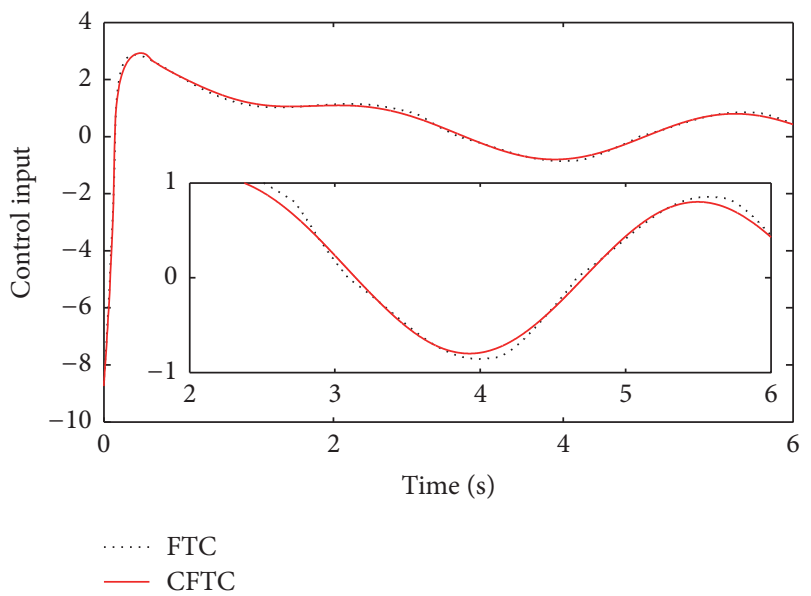

(d)

FIgURE 3: The response curves of system (43) under two different control FTC (44) (dotted line) and CFTC (45) (solid line). (a) State $x_{1}$, (b) state $x_{2}$, (c) state $z_{1}$, and (d) control input.

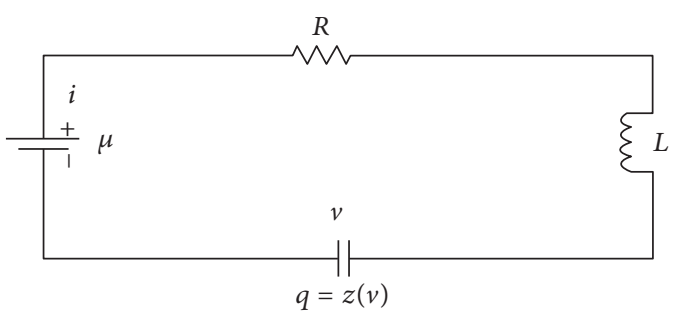

FIgURE 4: Nonlinear RLC circuit.

where $k_{1}, k_{2}, k_{3}$, and $k_{4}$ are appropriate positive constants, and $\gamma_{1}=1, \gamma_{i}=\gamma_{1}+(i-1) \tau_{3}, i=2,3,4$ with $\tau_{3} \in(-1 / 4,0)$.

The simulation is conducted $\left(x_{1}(0), x_{2}(0), z_{1}(0), y_{1}(0), y_{2}(0)\right.$, $\left.y_{3}(0), y_{4}(0)\right)=(2.5,-3,2,-3,0,0,0)$. The response curves of the closed-loop systems (48)-(49) are shown in Figure 5.

\section{Conclusion}

The problem of composite finite-time stabilization problem for a class of NDASs subject to external disturbance has been considered. Homogeneous system theory and the adding a power integrator technique have been adopted to the analysis and design of continuous finite-time disturbance observer and composite controller. It has been shown that the proposed composite controller not only can stabilize the NDAS with disturbance in finite time, but also has the nominal performance recovery ability. The validity of the proposed control approach has been demonstrated by two examples.

\section{Appendix}

\section{A. Preliminaries}

In this section, we introduce some definitions and lemmas which will be used throughout the paper.

Definition A.1 (see [28]). Consider a system

$$
\dot{x}=f(x), \quad f(0)=0, x \in \mathbb{R}^{n},
$$

where $f(\cdot): \mathbb{D} \rightarrow \mathbb{R}^{n}$ is non-Lipschitz continuous on an neighborhood $\mathbb{D}$ of the origin $x=0$ in $\mathbb{R}^{n}$. The equilibrium 


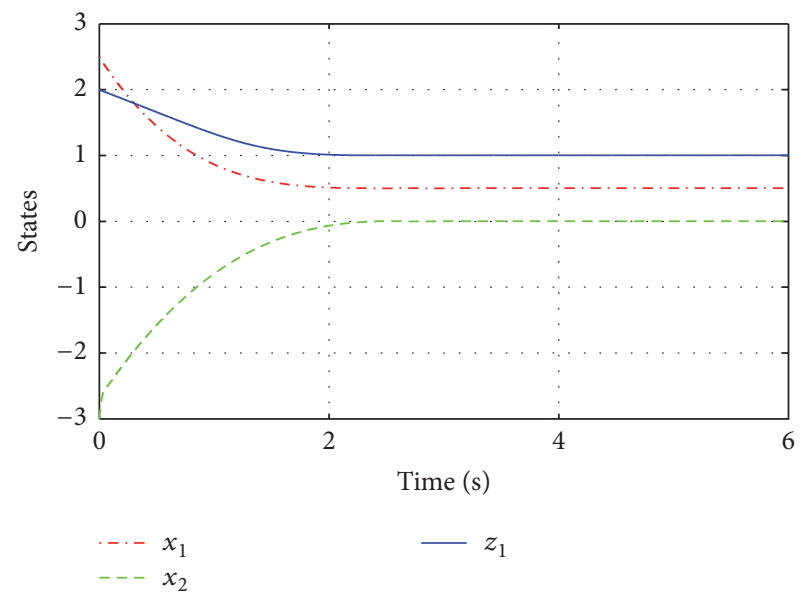

(a)

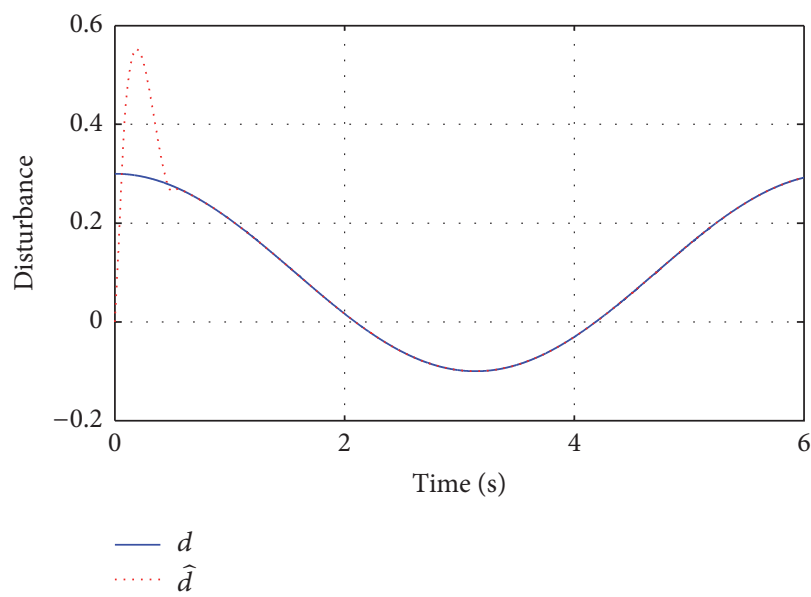

(b)

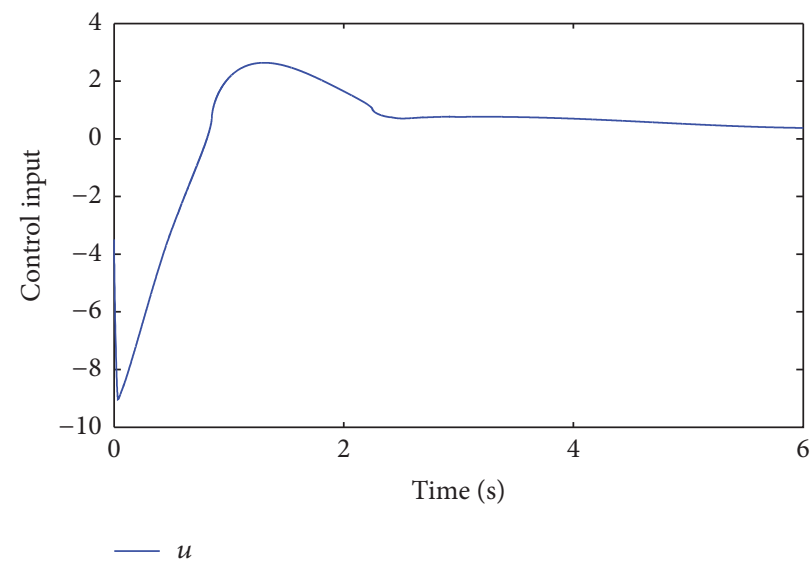

(c)

FIGURE 5: Response curves of the closed-loop systems (48)-(49).

$x=0$ of (A.1) is (locally) finite-time stable if there are an open neighborhood $\mathbb{U}$ of the origin and a function $T: \mathbb{U} \backslash\{0\} \rightarrow$ $[0, \infty)$, such that every solution trajectory $x\left(t, x_{0}\right)$ of system (A.1) starting from the initial point $x_{0} \in \mathbb{U} \backslash\{0\}$ is well defined and unique in forward time for $t \in\left[0, T\left(x_{0}\right)\right)$, and $\lim _{t \rightarrow T\left(x_{0}\right)} x\left(t, x_{0}\right)=0$. Here $T\left(x_{0}\right)$ is called the convergence time (of the initial state $x_{0}$ ). The equilibrium of system (A.1) is finite time stable if it is Lyapunov stable and finite-time convergent. If $\mathbb{U}=\mathbb{D}=\mathbb{R}^{n}$, the origin is a globally finite-time stable equilibrium.

Lemma A.2 (see [28]). Assume that there exists a continuous function $V(x): \mathbb{U} \rightarrow \mathbb{R}$ such that the following conditions hold:

(i) $V(x)$ is positive definite.

(ii) There exist real numbers $c>0$ and $\alpha \in(0,1)$ and an open neighborhood $\mathbb{U}_{0} \subset \mathbb{U}$ of the origin such that $\dot{V}(x)+c V^{\alpha}(x) \leq 0, x \in \mathbb{U}_{0} \backslash\{0\}$.

Then the origin is a finite-time stable equilibrium of system (A.1) and the finite convergence time $T$ satisfies $T \leq$ $V(x(0))^{1-\alpha} / c(1-\alpha)$. If $\mathbb{U}=\mathbb{U}_{0}=\mathbb{R}^{n}$ and $V(x)$ is proper, then the origin is a globally finite time stable equilibrium of (A.1).
Definition A.3 (see [42]). For fixed coordinates $\left(x_{1}, \ldots, x_{n}\right) \in$ $\mathbb{R}^{n}$ and real numbers $r_{i}>0, i=1, \ldots, n$,

(i) the dilation $\Delta_{\varepsilon}(x)=\left(\varepsilon^{r_{1}} x_{1}, \ldots, \varepsilon^{r_{n}} x_{n}\right), \forall \varepsilon>0$, with $r_{i}$ being called as the weights of the coordinates (for simplicity of notation, we define dilation weight $\Delta=$ $\left.\left(r_{1}, \ldots, r_{n}\right)\right)$;

(ii) a function $V \in \mathbb{C}\left(\mathbb{R}^{n}, \mathbb{R}\right)$ is said to be homogeneous of degree $\tau$ if there is a real number $\tau \in \mathbb{R}$ such that $\forall x \in \mathbb{R}^{n} \backslash\{0\}, \varepsilon>0, V\left(\Delta_{\varepsilon}(x)\right)=\varepsilon^{\tau} V\left(x_{1}, \ldots, x_{n}\right) ;$

(iii) a vector field $f \in \mathbb{C}\left(\mathbb{R}^{n}, \mathbb{R}^{n}\right)$ is said to be homogeneous of degree $\tau$ if there is a real number $\tau \in \mathbb{R}$ such that for $i=1, \ldots, n, \forall x \in \mathbb{R}^{n} \backslash\{0\}, \varepsilon_{i}>0, f_{i}\left(\Delta_{\varepsilon}(x)\right)=$ $\varepsilon^{\tau+r_{i}} f_{i}\left(x_{1}, \ldots, x_{n}\right)$;

(iv) a homogeneous $p$-norm is defined as $\|x\|_{\Delta, p}=$ $\left(\sum_{i=1}^{m}\left|x_{i}\right|^{p / r_{i}}\right)^{1 / p}, \forall x \in \mathbb{R}^{n}$, for a constant $p \geq 1$

Lemma A.4 (see [28]). Suppose $V: \mathbb{R}^{n} \rightarrow \mathbb{R}$ is a homogeneous function of degree $\tau$ with respect to the dilation weight $\Delta$. Then the following holds:

(i) $\partial V / \partial x_{i}$ is homogeneous of degree $\tau-r_{i}$ with $r_{i}$ being the homogeneous weight of $x_{i}$. 
(ii) There is a constant c such that $V(x) \leq c\|x\|_{\Delta}^{\tau}$. Moreover, if $V(x)$ is positive definite, $\underline{c}\|x\|_{\Delta}^{\tau} \leq V(x)$ for a constant $\underline{c}>0$.

Lemma A.5 (see [34]). The system

$$
\dot{z}=f(z, v), \quad z \in \mathbb{R}^{n}, v \in \mathbb{R}^{m}
$$

with $f(0,0)=0$, is finite-time input-to-state stable (FTISS), if it has an FTISS-Lyapunov function.

Lemma A.6 (see [45]). For any positive constants $c$, $d$ and any real-valued function $\eta(x, y)>0$, the following inequality holds:

$$
\begin{aligned}
|x|^{c}|y|^{d} \leq & \frac{c}{c+d} \eta(x, y)|x|^{c+d} \\
& +\frac{d}{c+d} \eta^{-c / d}(x, y)|y|^{c+d} .
\end{aligned}
$$

Lemma A.7 (see [38]). Let $p \in \mathbb{R}_{\text {odd }}^{\geq 1}$ and $x, y$ be real-valued functions; then, for a constant $c>0$ the following inequalities hold:

$$
\begin{aligned}
\left|x^{p}-y^{p}\right| & \leq p|x-y|\left(x^{p-1}+y^{p-1}\right) \\
& \leq c|x-y|\left|(x-y)^{p-1}+y^{p-1}\right| \\
\left|x^{1 / p}-y^{1 / p}\right| & \leq 2^{1-1 / p}|x-y|^{1 / p} .
\end{aligned}
$$

\section{B. The Proofs of Propositions}

The proof of Propositions 7 and 8 are given in this section. For the sake of simplicity, here we will use a generic constant $\underline{c}$ which exemplifies any limited positive constant value and $\underline{c}_{i}\left(\underline{x}_{i}\right)$ represents smooth, nonnegative function, either of which may be implicitly changed in different places.

Proof of Proposition 7. Note that $0<\left(r_{j}+\tau_{1}\right) / m_{j}<1$, $j=1, \ldots, i$; based on Assumption 4, inequality (7), and the famous inequality $(a+b)^{c} \leq a^{c}+b^{c}, \forall a \geq 0, b \geq 0, c \in(0,1)$, we have for $j=1, \ldots, i$

$$
\begin{aligned}
& \left|z_{j}\right|^{\left(r_{i}+\tau_{1}\right) / m_{j}} \leq b_{j}\left(\underline{x}_{j}\right)^{\left(r_{i}+\tau_{1}\right) / m_{j}}\left(\left|x_{1}\right|^{\left(r_{i}+\tau_{1}\right) / r_{1}}+\cdots\right. \\
& \quad+\left|x_{j}\right|^{\left(r_{j}+\tau_{1}\right) / r_{j}}+\left|z_{1}\right|^{\left(r_{i}+\tau_{1}\right) / m_{1}}+\cdots \\
& \left.\quad+\left|z_{j-1}\right|^{\left(r_{i}+\tau_{1}\right) / m_{j-1}}\right) .
\end{aligned}
$$

By using (B.1) and Assumption 2, it follows from (3) that

$$
\begin{aligned}
\left|\phi_{i}\right| & \leq c_{i}\left(\underline{x}_{i}\right)\left(\left|x_{1}\right|^{\left(r_{i}+\tau_{1}\right) / r_{1}}+\cdots+\left|x_{i}\right|^{\left(r_{i}+\tau_{1}\right) / r_{i}}\right. \\
& \left.+\left|z_{1}\right|^{\left(r_{i}+\tau_{1}\right) / m_{1}}+\left|z_{2}\right|^{\left(r_{i}+\tau_{1}\right) / m_{2}}+\cdots+\left|z_{i}\right|^{\left(r_{i}+\tau_{1}\right) / m_{i}}\right) \\
\leq & c_{i}\left(\underline{x}_{i}\right)\left(\left|x_{1}\right|^{\left(r_{i}+\tau_{1}\right) / r_{1}}+\cdots+\left|x_{i}\right|^{\left(r_{i}+\tau_{1}\right) / r_{i}}\right. \\
& +\left|z_{1}\right|^{\left(r_{i}+\tau_{1}\right) / m_{1}}+\left|z_{2}\right|^{\left(r_{i}+\tau_{1}\right) / m_{2}}+\cdots \\
& +\left|z_{i-1}\right|^{\left(r_{i}+\tau_{1}\right) / m_{i-1}}+b_{i}\left(\underline{x}_{i}\right)^{\left(r_{i}+\tau_{1}\right) / m_{i}}\left(\left|x_{1}\right|^{\left(r_{i}+\tau_{1}\right) / r_{1}}\right.
\end{aligned}
$$

$$
\begin{aligned}
& +\cdots+\left|x_{i}\right|^{\left(r_{j}+\tau_{1}\right) / r_{i}}+\left|z_{1}\right|^{\left(r_{i}+\tau_{1}\right) / m_{1}}+\cdots \\
& \left.\left.+\left|z_{i-1}\right|^{\left(r_{i}+\tau_{1}\right) / m_{i-1}}\right)\right) \\
\leq & \underline{c}_{i}\left(\underline{x}_{i}\right)\left(\left|x_{1}\right|^{\left(r_{i}+\tau_{1}\right) / r_{1}}+\cdots+\left|x_{i}\right|^{\left(r_{i}+\tau_{1}\right) / r_{i}}\right. \\
& +\left|z_{1}\right|^{\left(r_{i}+\tau_{1}\right) / m_{1}}+\left|z_{2}\right|^{\left(r_{i}+\tau_{1}\right) / m_{2}}+\cdots \\
& \left.+\left|z_{i-1}\right|^{\left(r_{i}+\tau_{1}\right) / m_{i-1}}\right)
\end{aligned}
$$

$$
\begin{aligned}
\leq & c_{i}\left(\underline{x}_{i}\right)\left(\left|x_{1}\right|^{\left(r_{i}+\tau_{1}\right) / r_{1}}+\cdots+\left|x_{i}\right|^{\left(r_{i}+\tau_{1}\right) / r_{i}}\right) \\
= & c_{i}\left(\underline{x}_{i}\right)\left(\left|\xi_{1}\right|^{r_{i}+\tau_{1}}+\left|\xi_{2}-\bar{\beta}_{1}\left(x_{1}\right) \xi_{1}\right|^{r_{i}+\tau_{1}}+\cdots+\mid \xi_{i}\right. \\
& \left.-\left.\bar{\beta}_{i-1}\left(\underline{x}_{i-1}\right) \xi_{i-1}\right|^{r_{i}+\tau_{1}}\right) \\
\leq & c_{i}\left(\underline{x}_{i}\right)\left(\left|\xi_{1}\right|^{r_{i}+\tau_{1}}+\left|\xi_{2}\right|^{r_{i}+\tau_{1}}+\cdots+\left|\xi_{i}\right|^{r_{i}+\tau_{1}}\right) .
\end{aligned}
$$

By (B.2) and Lemma A.6, it can be proved that there exists a $\mathbb{C}^{\infty}$ function $a_{3}\left(\underline{x}_{i}\right)>0$ such that

$$
\begin{aligned}
\xi_{i}^{2-\tau_{1}-r_{i}} \phi_{i}(\cdot) \leq c_{i}\left(\underline{x}_{i}\right)\left|\xi_{i}\right|^{2-\tau_{1}-r_{i}} \\
\cdot\left(\left|\xi_{1}\right|^{r_{i}+\tau_{1}}+\left|\xi_{2}\right|^{r_{i}+\tau_{1}}+\cdots+\left|\xi_{i}\right|^{r_{i}+\tau_{1}}\right) \\
\quad \leq \frac{1}{2}\left(\xi_{1}^{2}+\xi_{2}^{2}+\cdots+\xi_{i-2}^{2}\right)+\frac{1}{3} \xi_{i-1}^{2}+a_{3}\left(\underline{x}_{i}\right) \xi_{i}^{2} .
\end{aligned}
$$

Proof of Proposition 8. By the definition of $W_{i}$ and Lemma A.7, it can be shown that for $k=1, \ldots, i-1$

$$
\begin{aligned}
& \left|\frac{\partial W_{i}}{\partial x_{k}} \dot{x}_{k}\right|=\mid-\frac{2-\tau_{1}-r_{i}}{r_{i}} \frac{\partial x_{i}^{* 1 / r_{i}}}{\partial x_{k}} \\
& \cdot \int_{x_{i}^{*}}^{x_{i}}\left(s^{1 / r_{i}}-x_{i}^{* 1 / r_{i}}\right)^{1-\tau_{1}-r_{i}} d s \dot{x}_{k}|\leq \underline{c}| x_{i}-x_{i}^{*} \mid \\
& \cdot\left|\xi_{i}\right|^{1-\tau_{1}-r_{i}}\left|\frac{\partial x_{i}^{* 1 / r_{i}}}{\partial x_{k}} \dot{x}_{k}\right| \leq \underline{c}\left|\xi_{i}\right|^{1-\tau_{1}}\left|\frac{\partial x_{i}^{* 1 / r_{i}}}{\partial x_{k}} \dot{x}_{k}\right| .
\end{aligned}
$$

By the definition of $x_{i}^{*}$, we have $x_{i}^{* 1 / r_{i}}=\left(-\beta_{i-1}\left(\underline{x}_{i-1}\right)\right)^{1 / r_{i}}$. $\xi_{i-1}=\bar{\beta}_{i-1}\left(\underline{x}_{i-1}\right) \xi_{i-1}$. It follows that

$$
\begin{aligned}
\left|\frac{\partial x_{i}^{* 1 / r_{i}}}{\partial x_{k}}\right| & =\left|\frac{\partial \bar{\beta}_{i-1} \xi_{i-1}}{\partial x_{k}}\right|=\underline{c}_{i-1}\left(\underline{x}_{i-1}\right)\left|x_{k}^{\left(1-r_{k}\right) / r_{k}}\right| \\
& =\underline{c}_{i-1}\left(\underline{x}_{i-1}\right)\left|\xi_{k}+x_{k}^{*\left(1 / r_{k}\right)}\right|^{1-r_{k}} \\
& =\underline{c}_{i-1}\left(\underline{x}_{i-1}\right)\left|\xi_{k}+\bar{\beta}_{k-1}\left(\underline{x}_{k-1}\right) \xi_{k-1}\right|^{1-r_{k}} \\
& \leq \underline{c}_{i-1}\left(\underline{x}_{i-1}\right)\left(\left|\xi_{k}\right|^{1-r_{k}}+\left|\xi_{k-1}\right|^{1-r_{k}}\right) .
\end{aligned}
$$


By Assumptions 2 and 4, it is easy to know that $\dot{x}_{k}$ is homogeneous of degree $r_{k}+\tau_{1}$ with respect to the dilation weights $r=\left(r_{1}, \ldots, r_{i-1}\right)$. Based on Assumption 4, the definition of $\xi_{k}$ in (15), and Lemma A.6, it can be proved that

$$
\begin{aligned}
&\left|\dot{x}_{k}\right| \leq \underline{c}\left(\underline{x}_{k}\right)\left(\left|x_{1}\right|^{\left(r_{1}+\tau_{1}\right) / r_{1}}+\cdots+\left|x_{k}\right|^{\left(r_{1}+\tau_{1}\right) / r_{k}}\right. \\
&\left.+\left|z_{1}\right|^{\left(r_{1}+\tau_{1}\right) / m_{1}}+\cdots+\left|z_{k}\right|^{\left(r_{1}+\tau_{1}\right) / m_{k}}+\left|x_{k+1}\right|\right) \\
& \leq \underline{c}\left(\underline{x}_{k}\right)\left(\left|x_{1}\right|^{\left(r_{1}+\tau_{1}\right) / r_{1}}+\cdots+\left|x_{k}\right|^{\left(r_{1}+\tau_{1}\right) / r_{k}}\right. \\
&\left.+\left|x_{k+1}\right|\right)=\underline{c}\left(\underline{x}_{k}\right)\left(\left|\xi_{1}\right|^{r_{1}+\tau_{1}}+\cdots\right. \\
&+\left|\xi_{k}-\bar{\beta}_{k-1}\left(\underline{x}_{k-1}\right) \xi_{k-1}\right|^{r_{1}+\tau_{1}} \\
&\left.+\left|\xi_{k+1}-\bar{\beta}_{k}\left(\underline{x}_{k}\right) \xi_{k}\right|^{r_{1}+\tau_{1}}\right) \leq \underline{c}\left(\underline{x}_{k}\right)\left(\left|\xi_{1}\right|^{r_{1}+\tau_{1}}+\cdots\right. \\
&\left.+\left|\xi_{k}\right|^{r_{1}+\tau_{1}}+\left|\xi_{k+1}\right|^{r_{1}+\tau_{1}}\right) . \\
& \text { Combining }(\mathrm{B} .5) \text { with }(\mathrm{B} .6) \text { leads to } \\
&\left|\frac{\partial x_{i}^{* \mu / r_{i}}}{\partial x_{k}} \dot{x}_{k}\right| \leq \underline{c}_{i-1}\left(\underline{x}_{i-1}\right)\left(\left|\xi_{k}\right|^{1-r_{k}}+\left|\xi_{k-1}\right|^{1-r_{k}}\right) \underline{c}\left(\underline{x}_{k}\right) \\
& \quad \cdot\left(\left|\xi_{1}\right|^{r_{1}+\tau_{1}}+\cdots+\left|\xi_{k}\right|^{r_{1}+\tau_{1}}+\left|\xi_{k+1}\right|^{r_{1}+\tau_{1}}\right) \\
& \quad \leq \underline{c}_{i-1}\left(\underline{x}_{i-1}\right) \\
& \quad \cdot\left(\left|\xi_{1}\right|^{1+\tau_{1}}+\left|\xi_{2}\right|^{1+\tau_{1}}+\cdots+\left|\xi_{k}\right|^{1+\tau_{1}}+\left|\xi_{k+1}\right|^{1+\tau_{1}}\right) .
\end{aligned}
$$

By Lemma A.6, (B.4) together with (B.7) gives

$$
\begin{aligned}
& \left|\frac{\partial W_{i}}{\partial x_{k}} \dot{x}_{k}\right| \leq \underline{c}\left|\xi_{i}\right|^{1-\tau_{1}} \underline{c}_{i-1}\left(\underline{x}_{i-1}\right) \\
& \cdot\left(\left|\xi_{1}\right|^{1+\tau_{1}}+\left|\xi_{2}\right|^{1+\tau_{1}}+\cdots+\left|\xi_{k}\right|^{1+\tau_{1}}+\left|\xi_{k+1}\right|^{1+\tau_{1}}\right) \\
& \quad \leq \frac{1}{2}\left(\xi_{1}^{2}+\xi_{2}^{2}+\cdots+\xi_{i-2}^{2}\right)+\frac{1}{3} \xi_{i-1}^{2}+a_{2}\left(\underline{x}_{i}\right) \xi_{i}^{2}
\end{aligned}
$$

where $a_{2}\left(\underline{x}_{i}\right)$ is a smooth strictly positive function.

\section{Competing Interests}

The authors declare that they have no competing interests.

\section{Acknowledgments}

This work is supported by the Natural Science Foundation of China (61540068 and 61503122), Foundation of Henan Department of Science and Technology (162300410087), and Foundation of Henan Education Committee (12B120004).

\section{References}

[1] R. Riaza, "Double SIB points in differential-algebraic systems," IEEE Transactions on Automatic Control, vol. 48, no. 9, pp. 16251629, 2003.
[2] D. J. Hill and I. M. Mareels, "Stability theory for differential/algebraic systems with application to power systems," Institute of Electrical and Electronics Engineers. Transactions on Circuits and Systems, vol. 37, no. 11, pp. 1416-1423, 1990.

[3] X. Liu and D. W. Ho, "Stabilization of non-linear differentialalgebraic equation systems," International Journal of Control, vol. 77, no. 7, pp. 671-684, 2004.

[4] L. Dai, Singular Control Systems, vol. 118 of Lecture Notes in Control and Information Sciences, Springer, Berlin, 1989.

[5] S. Y. Xu and J. Lam, Robust Control and Filtering of Singular Systems, Springer, Berlin, Germany, 2006.

[6] Q. Lan, Y. Liu, H. Niu, and J. Liang, "Robust reliable guaranteed cost control for uncertain singular systems with time-delay," Journal of Systems Engineering and Electronics, vol. 21, no. 1, pp. 110-117, 2010

[7] L. Xiaoping and S. Celikovsky, "Feedback control of affine nonlinear singular control systems," International Journal of Control, vol. 68, no. 4, pp. 753-774, 1997.

[8] C. Yang, J. Sun, Q. Zhang, and X. Ma, "Lyapunov stability and strong passivity analysis for nonlinear descriptor systems," IEEE Transactions on Circuits and Systems. I. Regular Papers, vol. 60, no. 4, pp. 1003-1012, 2013.

[9] L. Sun, G. Feng, and Y. Wang, "Finite-time stabilization and Ho control for a class of nonlinear Hamiltonian descriptor systems with applications to affine nonlinear descriptor systems," Automatica, vol. 50, no. 8, pp. 2090-2097, 2014.

[10] H.-S. Wang, C.-F. Yung, and F.-R. Chang, "Ho control for nonlinear descriptor systems," Institute of Electrical and Electronics Engineers. Transactions on Automatic Control, vol. 47, no. 11, pp. 1919-1925, 2002.

[11] F. Castaños, D. Hernández, and L. Fridman, "Integral slidingmode control for linear time-invariant implicit systems," Automatica, vol. 50, no. 3, pp. 971-975, 2014.

[12] J. Xu and J. Sun, "Finite-time stability of linear time-varying singular impulsive systems," IET Control Theory \&amp; Applications, vol. 4, no. 10, pp. 2239-2244, 2010.

[13] J. L. Lin and S. J. Chen, "Robustness analysis of uncertain linear singular systems with output feedback control," Institute of Electrical and Electronics Engineers. Transactions on Automatic Control, vol. 44, no. 10, pp. 1924-1929, 1999.

[14] D. Lin and W. Lan, "Output feedback composite nonlinear feedback control for singular systems with input saturation," Journal of the Franklin Institute, vol. 352, no. 1, pp. 384-398, 2015.

[15] N. Harris McClamroch, "Feedback stabilization of control systems described by a class of nonlinear differential-algebraic equations," Systems \& Control Letters, vol. 15, no. 1, pp. 53-60, 1990.

[16] D. F. Coutinho, A. S. Bazanella, A. Trofino, and A. S. e Silva, "Stability analysis and control of a class of differentialalgebraic nonlinear systems," International Journal of Robust and Nonlinear Control, vol. 14, no. 16, pp. 1301-1326, 2004.

[17] Z. P. Jiang and M. Ikeda, "Backstepping design for stabilization of nonlinear differential-algebraic systems," in Proceedings of the 48th Japan Joint Automatic Control Conference, pp. 927-930, Nagano-ken, Japan, November 2005.

[18] W.-H. Chen, "Harmonic disturbance observer for nonlinear systems," Journal of Dynamic Systems, Measurement and Control, vol. 125, no. 1, pp. 114-117, 2003.

[19] L. Marconi and A. Teel, "Matched disturbance suppression for nonlinear systems stabilizable by logic-based feedback," Automatica, vol. 48, no. 5, pp. 886-893, 2012. 
[20] K.-S. Kim, K.-H. Rew, and S. Kim, "Disturbance observer for estimating higher order disturbances in time series expansion," IEEE Transactions on Automatic Control, vol. 55, no. 8, pp. 19051911, 2010.

[21] Y. Z. Tsypkin, J. D. Mason, E. D. Avedyan, K. Warwick, and I. K. Levin, "Neural networks for identification of nonlinear systems under random piecewise polynomial disturbances," IEEE Transactions on Neural Networks, vol. 10, no. 2, pp. 303312, 1999.

[22] J. Yang, S. Li, J. Su, and X. Yu, "Continuous nonsingular terminal sliding mode control for systems with mismatched disturbances," Automatica, vol. 49, no. 7, pp. 2287-2291, 2013.

[23] J. Han, "From PID to active disturbance rejection control," IEEE Transactions on Industrial Electronics, vol. 56, no. 3, pp. 900906, 2009.

[24] Y. Liu, C. Li, and R. Wu, "Feedback control of nonlinear differential algebraic systems using Hamiltonian function method," Science in China. Series F. Information Sciences, vol. 49, no. 4, pp. 436-445, 2006.

[25] Z. Chen and J. Huang, "Solution of output regulation of singular nonlinear systems by normal output feedback," IEEE Transactions on Automatic Control, vol. 47, no. 5, pp. 808-813, 2002.

[26] S. Pang, J. Huang, and Y. Bai, "Robust output regulation of singular nonlinear systems via a nonlinear internal model," IEEE Transactions on Automatic Control, vol. 50, no. 2, pp. 222228, 2005.

[27] X. M. Yao, L. Q. Zhu, and L. Guo, “Disturbance-observer-based control $\& H_{\infty}$ control for non-linear Markovian jump singular systems with multiple disturbances," IET Control Theory \& Applications, vol. 8, no. 16, pp. 1689-1697, 2014.

[28] S. P. Bhat and D. S. Bernstein, "Continuous finite-time stabilization of the translational and rotational double integrators," IEEE Transactions on Automatic Control, vol. 43, no. 5, pp. 678-682, 1998.

[29] S. Ding, J. Wang, and W. X. Zheng, "Second-order sliding mode control for nonlinear uncertain systems bounded by positive functions," IEEE Transactions on Industrial Electronics, vol. 62, no. 9, pp. 5899-5909, 2015.

[30] S. Ding, A. Levant, and S. Li, "Simple homogeneous slidingmode controller," Automatica, vol. 67, pp. 22-32, 2016.

[31] Z.-Y. Sun, L.-R. Xue, and K. M. Zhang, "A new approach to finite-time adaptive stabilization of high-order uncertain nonlinear system," Automatica, vol. 58, no. 8, pp. 60-66, 2015.

[32] A. Levant, "Higher-order sliding modes, differentiation and output-feedback control," International Journal of Control, vol. 76, no. 9-10, pp. 924-941, 2003.

[33] X. Huang, W. Lin, and B. Yang, "Global finite-time stabilization of a class of uncertain nonlinear systems," Automatica. A Journal of IFAC, the International Federation of Automatic Control, vol. 41, no. 5, pp. 881-888, 2005.

[34] Y. Hong, Z.-P. Jiang, and G. Feng, "Finite-time input-to-state stability and applications to finite-time control design," SIAM Journal on Control and Optimization, vol. 48, no. 7, pp. 43954418, 2010.

[35] J. Li, C. Qian, and S. Ding, "Global finite-time stabilisation by output feedback for a class of uncertain nonlinear systems," International Journal of Control, vol. 83, no. 11, pp. 2241-2252, 2010.

[36] S. Li, H. Du, and X. Lin, "Finite-time consensus algorithm for multi-agent systems with double-integrator dynamics," Automatica, vol. 47, no. 8, pp. 1706-1712, 2011.
[37] H. Du, C. Qian, S. Yang, and S. Li, "Recursive design of finitetime convergent observers for a class of time-varying nonlinear systems," Automatica. A Journal of IFAC, the International Federation of Automatic Control, vol. 49, no. 2, pp. 601-609, 2013.

[38] Q. Lan, S. Li, S. Khoo, and P. Shi, "Global finite-time stabilisation for a class of stochastic nonlinear systems by output feedback," International Journal of Control, vol. 88, no. 3, pp. 494-506, 2015.

[39] H. Sun and L. Guo, "Neural network-based dobc for a class of nonlinear systems with unmatched disturbances," IEEE Transactions on Neural Networks and Learning Systems, 2016.

[40] Y. Li, H. Sun, G. Zong, and L. Hou, "Disturbance-observerbased-control and L2-Lo resilient control for Markovian jump non-linear systems with multiple disturbances and its application to single robot arm system," IET Control Theory \& Applications, vol. 10, no. 2, pp. 226-233, 2016.

[41] W. Lin and C. Qian, "Adding one power integrator: a tool for global stabilization of high-order lower-triangular systems," Systems \& Control Letters, vol. 39, no. 5, pp. 339-351, 2000.

[42] L. Rosier, "Homogeneous Lyapunov function for homogeneous continuous vector field," Systems \& Control Letters, vol. 19, no. 6, pp. 467-473, 1992.

[43] A. E. Assoudi, E. H. E. Yaagoubi, and H. Hammouri, "A high gain observer for a class of implicit systems," in Proceedings of the 44th IEEE Conference on Decision and Control, and the European Control Conference, pp. 6359-6363, Seville, Spain, December 2005.

[44] L. Sun and Y. Wang, "An undecomposed approach to control design for a class of nonlinear descriptor systems," International Journal of Robust and Nonlinear Control, vol. 23, no. 6, pp. 695708, 2013.

[45] C. Qian and W. Lin, "A continuous feedback approach to global strong stabilization of nonlinear systems," IEEE Transactions on Automatic Control, vol. 46, no. 7, pp. 1061-1079, 2001.

[46] J. Polendo and C. Qian, "An expanded method to robustly stabilize uncertain nonlinear systems," Communications in Information and Systems, vol. 8, no. 1, pp. 55-70, 2008. 


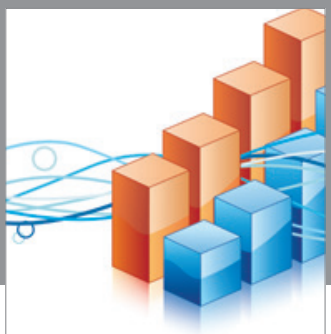

Advances in

Operations Research

vatem alat4

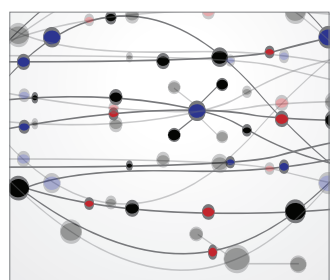

\section{The Scientific} World Journal
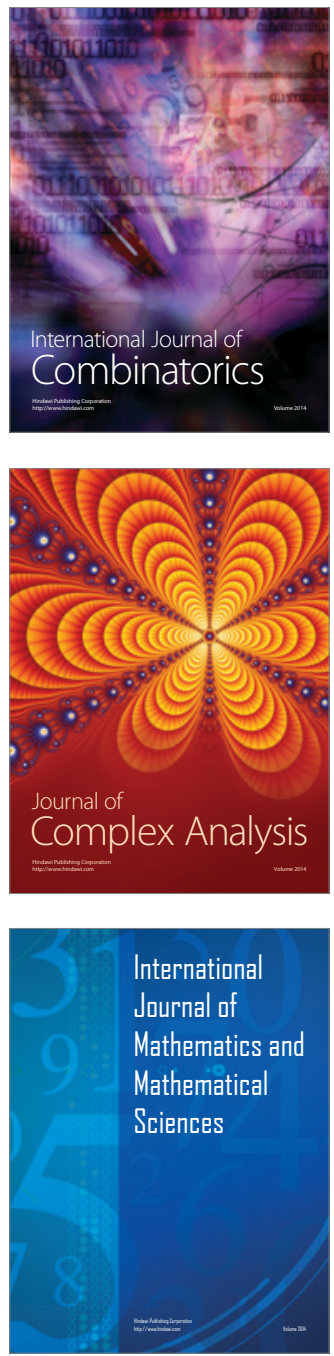
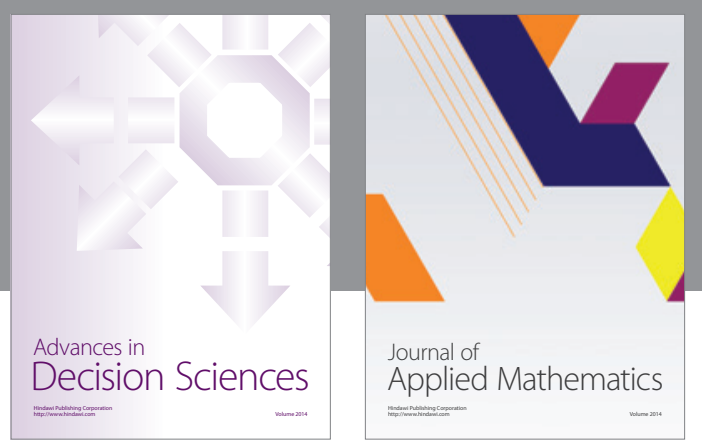

Algebra

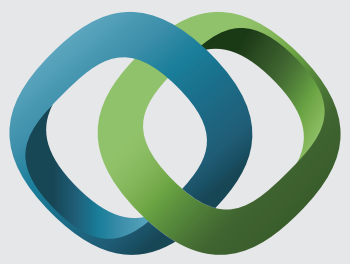

\section{Hindawi}

Submit your manuscripts at

https://www.hindawi.com
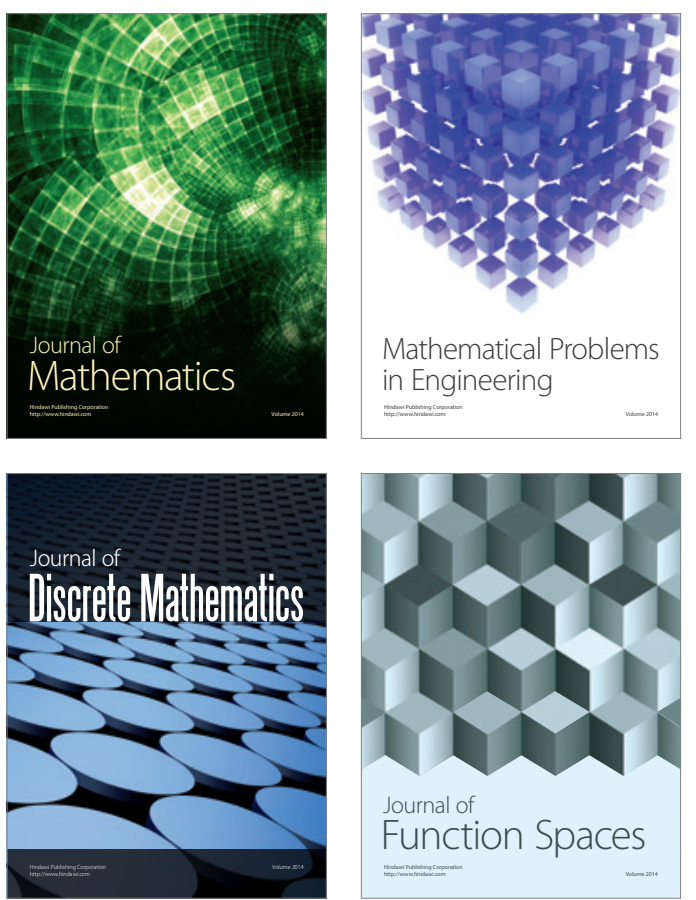

Mathematical Problems in Engineering
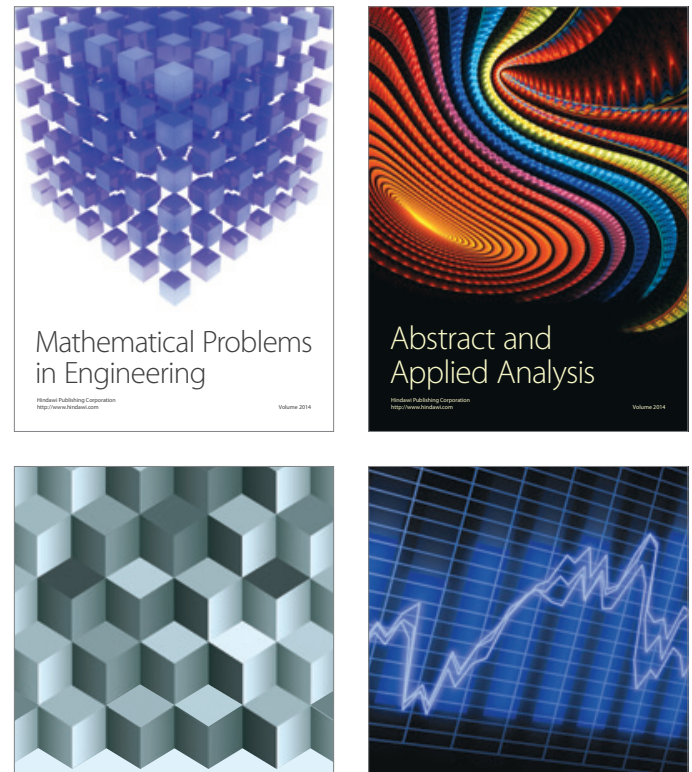

Journal of

Function Spaces

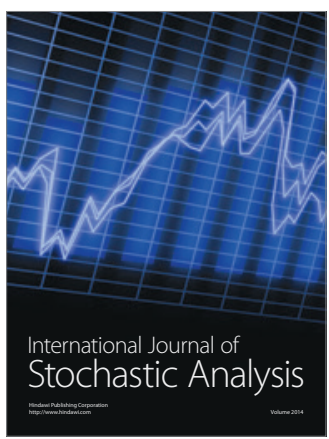

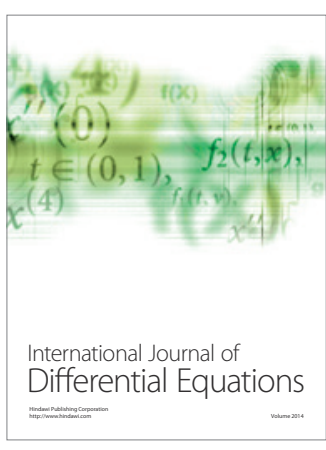
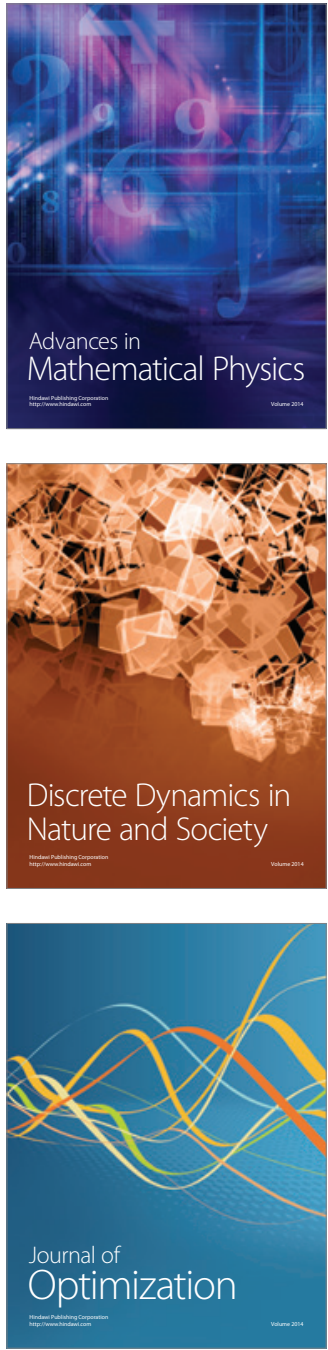